\title{
Wigner distributions and orbital angular momentum of a proton
}

\author{
D. Chakrabarti ${ }^{1, a}$, T. Maji ${ }^{1}$, C. Mondal ${ }^{1}$, A. Mukherjee ${ }^{2}$ \\ ${ }^{1}$ Department of Physics, Indian Institute of Technology Kanpur, Kanpur 208016, India \\ 2 Department of Physics, Indian Institute of Technology Bombay, Mumbai 400076, India
}

Received: 23 June 2016 / Accepted: 8 July 2016 / Published online: 20 July 2016

(C) The Author(s) 2016. This article is published with open access at Springerlink.com

\begin{abstract}
The Wigner distributions for the $u$ and the $d$ quarks in a proton are calculated using the light-front wave functions of the scalar quark-diquark model for a nucleon constructed from the soft-wall AdS/QCD correspondence. We present a detailed study of the quark orbital angular momentum and its correlation with the quark spin and the proton spin. The quark density distributions, considering the different polarizations of quarks and proton, in transverse momentum plane as well as in transverse impact parameter plane are presented for both $u$ and $d$ quarks.
\end{abstract}

\section{Introduction}

A complete understanding of the partonic structure of the nucleon is one of the challenging tasks in particle physics. Both theoretical and experimental efforts are going on to unravel the three-dimensional distributions of the partons and their contributions to the nucleon spin and angular momentum. Because of the nonperturbative nature of $\mathrm{QCD}$, it is very difficult to perform first principle calculations of the hadron properties. However, a perturbative approach in the lightcone framework allows us to calculate the parton distribution function (PDF), $f(x)$, which gives the probability of having a parton with light-cone longitudinal momentum fraction $x$ inside a nucleon, but it contains no information as regards the transverse structure or angular momentum distributions. The spin correlations of partons are described by the helicity distribution, $g_{1}(x)$, and the transversity distributions, $h_{1}(x)$. The generalized parton distributions (GPDs) and the transverse momentum dependent distributions (TMDs) encode informations about the three-dimensional structure of the nucleons. In deeply virtual Compton scattering (DVCS), deeply virtual meson electroproduction (DVMPs), a more general views of parton distributions, in the collinear frame, is studied by GPDs [1-4] which are functions of longitudinal momen-

\footnotetext{
a e-mail: dipankar@iitk.ac.in
}

tum and two transverse impact parameter coordinates. TMDs [5-8] are functions of the transverse momentum of the parton and appear in semi-inclusive deep inelastic scattering (SIDIS) where the collinear picture is no longer sufficient to explain the single spin asymmetry (SSA).

Wigner distributions are six-dimensional distributions containing more general informations about the nucleon structure. Wigner distributions do not have a probabilistic interpretation, but in certain limits they reduce to GPDs and TMDs. The Wigner distributions are defined as functions of three momenta and three positions of a parton inside a nucleon. The concept of Wigner distributions was first introduced in $[9,10]$. In [11], five-dimensional Wigner distributions were proposed in the light-front formalism with three momentum and two position components of a parton. Wigner distributions integrated over transverse momentum give the GPDs at zero skewness, the TMDs are obtained by integrating over transverse impact parameters with zero momentum transfer and the integration over transverse momentum and transverse positions provide the PDFs. The Wigner distributions after integrating over the light-cone energy of the parton are interpreted as a Fourier transform of the corresponding generalized transverse momentum dependent distributions (GTMDs), which are functions of the light-cone threemomentum of the parton as well as the momentum transfer to the nucleon. The angular momentum of a quark is extracted from the Wigner distributions taking the phase space average. The spin-spin and spin-orbital angular momentum (OAM) correlations between a nucleon and a quark inside the nucleon can also be described from a phase space average of Wigner distributions. Wigner distributions have been studied in different models, e.g., in the light-cone constituent quark model [12-17], in the chiral soliton model [18-22], the light-front dressed quark model [23,24], and the light-cone spectator model [25]. In this work, we investigate the Wigner distributions for unpolarized and polarized proton and the orbital angular momentum (OAM) and spin-spin and spin-OAM correlations in a scalar diquark model of the proton [26] with 
the light-front wavefunctions modeled from AdS/QCD prediction.

The paper is organized as follows. We first introduce the light-front scalar diquark model in Sect. 2 and the Wigner distributions in Sect. 3. The different definitions of the orbital angular momentum are discussed in Sect. 4. Then in Sect. 5 , both analytical and numerical results in our model are discussed in detail. The correlation between the quark and proton spins and quark spin and OAM correlations are discussed in Sect. 6. The results are also compared with other models. The GTMDs in this model are briefly discussed in Sect. 7, and finally we conclude in Sect. 8 .

\section{Light-front diquark model}

In the diquark spectator model, one of the three valence quarks interacts with an external photon and other two valence quarks are considered as a diquark state of spin 0 (scalar diquark) or spin 1 (vector diquark). Therefore the proton state $|P ; S\rangle$ can be treated as a two-particle state in the Fock-state expansion. In this paper we consider the scalar diquark model developed in $[26,27]$. The average light-front momentum of the scalar diquark is $P_{X}=$ $\left((1-x) P^{+}, P_{X}^{-},-\mathbf{p}_{\perp}\right)$, where $x$ is the longitudinal momentum fraction carried by the struck quark.

The two-particle Fock-state expansions for $J^{z}= \pm \frac{1}{2}$ are given by

$$
\begin{aligned}
|P ; \pm\rangle= & \sum_{q} \int \frac{\mathrm{d} x \mathrm{~d}^{2} \mathbf{p}_{\perp}}{2(2 \pi)^{3} \sqrt{x(1-x)}} \\
& \times\left[\psi_{q+}^{ \pm}\left(x, \mathbf{p}_{\perp}\right)\left|+\frac{1}{2}, 0 ; x P^{+}, \mathbf{p}_{\perp}\right\rangle\right. \\
& \left.+\psi_{q-}^{ \pm}\left(x, \mathbf{p}_{\perp}\right)\left|-\frac{1}{2}, 0 ; x P^{+}, \mathbf{p}_{\perp}\right\rangle\right],
\end{aligned}
$$

where $\left|\lambda_{q}, \lambda_{s} ; x P^{+}, \mathbf{p}_{\perp}\right\rangle$ represents a two-particle state with a quark of helicity $\lambda_{q}$, and a diquark (spectator) of helicity $\lambda_{s}$. The $x P^{+}$and $\mathbf{p}_{\perp}$ are the longitudinal momentum and transverse momentum of the active quark, respectively. The $\psi_{q \lambda_{q}}^{\lambda_{N}}$ are the light-front wave functions corresponding to the nucleon helicity $\lambda_{N}= \pm$ and quark helicity $\lambda_{q}= \pm$. We adopt the generic ansatz for the quark-diquark model of the valence Fock state of the nucleon LFWFs [26], assuming a vanishing quark mass,

$\psi_{q+}^{+}\left(x, \mathbf{p}_{\perp}\right)=\phi_{q}^{(1)}\left(x, \mathbf{p}_{\perp}\right)$,

$\psi_{q-}^{+}\left(x, \mathbf{p}_{\perp}\right)=-\frac{p^{1}+i p^{2}}{x M} \phi_{q}^{(2)}\left(x, \mathbf{p}_{\perp}\right)$, $\psi_{q+}^{-}\left(x, \mathbf{p}_{\perp}\right)=\frac{p^{1}-i p^{2}}{x M} \phi_{q}^{(2)}\left(x, \mathbf{p}_{\perp}\right)$,

$\psi_{q-}^{-}\left(x, \mathbf{p}_{\perp}\right)=\phi_{q}^{(1)}\left(x, \mathbf{p}_{\perp}\right)$,

where $\varphi_{q}^{(1)}\left(x, \mathbf{p}_{\perp}\right)$ and $\varphi_{q}^{(2)}\left(x, \mathbf{p}_{\perp}\right)$ are the wave functions predicted by soft-wall AdS/QCD in [28] with the AdS/QCD scale parameter $\kappa=0.4 \mathrm{GeV}$,

$$
\begin{aligned}
\varphi_{q}^{(i)}\left(x, \mathbf{p}_{\perp}\right)= & N_{q}^{(i)} \frac{4 \pi}{\kappa} \sqrt{\frac{\log (1 / x)}{1-x}} x^{(i)}(1-x)^{b_{q}^{(i)}} \\
& \times \exp \left[-\frac{\mathbf{p}_{\perp}^{2}}{2 \kappa^{2}} \frac{\log (1 / x)}{(1-x)^{2}}\right] .
\end{aligned}
$$

The values of the parameters $a_{q}^{(i)}, b_{q}^{(i)}$, and $N_{q}^{(i)}$ are fixed in $[29,30]$ by fitting the nucleon form-factor data. For completeness, we list the parameters in Table 1. This is a very simplistic model of the proton. It describes the proton by a scalar diquark and a quark and does not assume the $S U(4)$ symmetry of the usual diquark models [31] where both scalar and axial vector diquarks are considered.

\section{Wigner distribution}

In the light-front framework, the five-dimensional Wigner distribution is defined as [32-34]

$\rho^{q[\Gamma]}\left(\mathbf{b}_{\perp}, \mathbf{p}_{\perp}, x ; S\right)=\int \frac{\mathrm{d}^{2} \Delta_{\perp}}{(2 \pi)^{2}} e^{-i \Delta_{\perp} . b_{\perp}} W^{q[\Gamma]}\left(\Delta_{\perp}, \mathbf{p}_{\perp}, x ; S\right)$

where the correlator $W^{[\Gamma]}$, at $\Delta^{+}=0$ and fixed light-cone time $z^{+}=0$, is given by [11]

$$
\begin{aligned}
& W^{q[\Gamma]}\left(\Delta_{\perp}, \mathbf{p}_{\perp}, x ; S\right)=\frac{1}{2} \int \frac{\mathrm{d} z^{-}}{(2 \pi)} \frac{\mathrm{d}^{2} z_{T}}{(2 \pi)^{2}} e^{i p . z} \\
& \left.\left\langle P^{\prime \prime} ; S\left|\bar{\psi}^{q}(-z / 2) \Gamma \mathcal{W}_{[-z / 2, z / 2]} \psi^{q}(z / 2)\right| P^{\prime} ; S\right\rangle\right|_{z^{+}=0}
\end{aligned}
$$

with the Dirac structure $\Gamma$, e.g., $\gamma^{+}, \gamma^{+} \gamma^{5}$. The $P^{\prime}=$ $\left(P^{+}, P^{\prime-}, \frac{\Delta_{\perp}}{2}\right)$ and the $P^{\prime \prime}=\left(P^{+}, P^{\prime \prime}--\frac{\Delta_{\perp}}{2}\right)$ are the initial and final momentum of proton. $W^{[\Gamma]}$ depends on the average momentum $P=\frac{1}{2}\left(P^{\prime \prime}+P^{\prime}\right)$ of the proton, the average quark momentum $\mathbf{p}_{\perp}=\frac{1}{2}\left(\mathbf{p}_{\perp}^{\prime \prime}+\mathbf{p}_{\perp}^{\prime}\right)$, the proton helicity $S$, and the transverse momentum transfer to the proton $\Delta_{\perp}=\left(P_{\perp}^{\prime \prime}-P_{\perp}^{\prime}\right)$. The Wilson line $\mathcal{W}_{[-z / 2, z / 2]}$ ensures the gauge invariance of the operator. We choose the symmetric frame where the components of the four-momenta, with
Table 1 The parameters in the light-front diquark model for $\kappa=0.4 \mathrm{GeV}$

\begin{tabular}{lllllll}
\hline Parameters & $a^{(1)}$ & $a^{(2)}$ & $b^{(1)}$ & $b^{(2)}$ & $N^{(1)}$ & $N^{(2)}$ \\
\hline$u$ quark & 0.02 & 1.05 & 0.022 & -0.15 & 2.055 & 1.322 \\
$d$ quark & 0.1 & 1.07 & 0.38 & -0.2 & 1.7618 & -2.4827 \\
\hline
\end{tabular}


skewness $\xi=0$, are

$P=\left[P^{+}, P^{-}, \mathbf{0}_{\perp}\right]$,

$p=\left[x P^{+}, p^{-}, \mathbf{p}_{\perp}\right]$,

$\Delta=\left[0,0, \Delta_{\perp}\right]$,

with $P^{-}=\frac{1}{2}\left(P^{\prime \prime-}+P^{\prime-}\right)=\frac{4 M^{2}+\Delta_{\perp}^{2}}{4 P^{+}}$(we use the notation $v^{ \pm}=v^{0} \pm v^{3}$ ). We calculate the matrix element of Eq. (5) in the scalar diquark model using the wave functions predicted by soft-wall AdS/QCD. The Wigner distributions, with the proton helicity $\Lambda$ and the quark helicity $\lambda$, for unpolarized and longitudinally polarized proton, are defined as

$$
\begin{aligned}
\rho_{\Lambda \lambda}^{q}\left(\mathbf{b}_{\perp}, \mathbf{p}_{\perp}, x\right)= & \frac{1}{2}\left[\rho^{q\left[\gamma^{+}\right]}\left(\mathbf{b}_{\perp}, \mathbf{p}_{\perp}, x ; \Lambda \hat{S}_{z}\right)\right. \\
& \left.+\lambda \rho^{q\left[\gamma^{+} \gamma^{5}\right]}\left(\mathbf{b}_{\perp}, \mathbf{p}_{\perp}, x ; \Lambda \hat{S}_{z}\right)\right],
\end{aligned}
$$

which can be decomposed as

$$
\begin{aligned}
\rho_{\Lambda \lambda}^{q}\left(\mathbf{b}_{\perp}, \mathbf{p}_{\perp}, x\right)= & \frac{1}{2}\left[\rho_{U U}^{q}\left(\mathbf{b}_{\perp}, \mathbf{p}_{\perp}, x\right)\right. \\
& +\Lambda \rho_{L U}^{q}\left(\mathbf{b}_{\perp}, \mathbf{p}_{\perp}, x\right)+\lambda \rho_{U L}^{q}\left(\mathbf{b}_{\perp}, \mathbf{p}_{\perp}, x\right) \\
& \left.+\Lambda \lambda \rho_{L L}^{q}\left(\mathbf{b}_{\perp}, \mathbf{p}_{\perp}, x\right)\right],
\end{aligned}
$$

corresponding to the proton spin $\Lambda=\uparrow, \downarrow$ and quark spin $\lambda=\uparrow, \downarrow$ (where $\uparrow$ and $\downarrow$ correspond to +1 and -1 , respectively). Here the Wigner distribution $\rho_{U U}^{q}\left(\mathbf{b}_{\perp}, \mathbf{p}_{\perp}, x\right)$ of the unpolarized quarks in an unpolarized proton, and the distortions $\rho_{L U}^{q}\left(\mathbf{b}_{\perp}, \mathbf{p}_{\perp}, x\right)$ due to unpolarized quarks in a longitudinally polarized proton, $\rho_{U L}^{q}\left(\mathbf{b}_{\perp}, \mathbf{p}_{\perp}, x\right)$ due to longitudinally polarized quarks in an unpolarized proton, and $\rho_{L L}^{q}\left(\mathbf{b}_{\perp}, \mathbf{p}_{\perp}, x\right)$ due to longitudinally polarized quark in a longitudinally polarized proton, are defined as

$$
\begin{aligned}
\rho_{U U}^{q}\left(\mathbf{b}_{\perp}, \mathbf{p}_{\perp}, x\right)= & \frac{1}{2}\left[\rho^{q\left[\gamma^{+}\right]}\left(\mathbf{b}_{\perp}, \mathbf{p}_{\perp}, x ;+\hat{S}_{z}\right)\right. \\
& \left.+\rho^{q\left[\gamma^{+}\right]}\left(\mathbf{b}_{\perp}, \mathbf{p}_{\perp}, x ;-\hat{S}_{z}\right)\right], \\
\rho_{L U}^{q}\left(\mathbf{b}_{\perp}, \mathbf{p}_{\perp}, x\right)= & \frac{1}{2}\left[\rho^{q\left[\gamma^{+}\right]}\left(\mathbf{b}_{\perp}, \mathbf{p}_{\perp}, x ;+\hat{S}_{z}\right)\right. \\
& \left.-\rho^{q\left[\gamma^{+}\right]}\left(\mathbf{b}_{\perp}, \mathbf{p}_{\perp}, x ;-\hat{S}_{z}\right)\right], \\
\rho_{U L}^{q}\left(\mathbf{b}_{\perp}, \mathbf{p}_{\perp}, x\right)= & \frac{1}{2}\left[\rho^{q\left[\gamma^{+} \gamma^{5}\right]}\left(\mathbf{b}_{\perp}, \mathbf{p}_{\perp}, x ;+\hat{S}_{z}\right)\right. \\
& \left.+\rho^{q\left[\gamma^{+} \gamma^{5}\right]}\left(\mathbf{b}_{\perp}, \mathbf{p}_{\perp}, x ;-\hat{S}_{z}\right)\right], \\
\rho_{L L}^{q}\left(\mathbf{b}_{\perp}, \mathbf{p}_{\perp}, x\right)= & \frac{1}{2}\left[\rho^{q\left[\gamma^{+} \gamma^{5}\right]}\left(\mathbf{b}_{\perp}, \mathbf{p}_{\perp}, x ;+\hat{S}_{z}\right)\right. \\
& \left.-\rho^{q\left[\gamma^{+} \gamma^{5}\right]}\left(\mathbf{b}_{\perp}, \mathbf{p}_{\perp}, x ;-\hat{S}_{z}\right)\right] .
\end{aligned}
$$

These four distributions are related with the Fourier transforms of the GTMDs as

$$
\begin{aligned}
\rho_{U U}^{q}\left(\mathbf{b}_{\perp}, \mathbf{p}_{\perp}, x\right)= & \mathcal{F}_{1,1}^{q}\left(x, 0, \mathbf{p}_{\perp}^{2}, \mathbf{p}_{\perp} \cdot \mathbf{b}_{\perp}, \mathbf{b}_{\perp}^{2}\right), \\
\rho_{L U}^{q}\left(\mathbf{b}_{\perp}, \mathbf{p}_{\perp}, x\right)= & -\frac{1}{M^{2}} \epsilon_{\perp}^{i j} p_{\perp}^{i} \\
& \times \frac{\partial}{\partial b_{\perp}^{j}} \mathcal{F}_{1,4}^{q}\left(x, 0, \mathbf{p}_{\perp}^{2}, \mathbf{p}_{\perp} \cdot \mathbf{b}_{\perp}, \mathbf{b}_{\perp}^{2}\right), \\
\rho_{U L}^{q}\left(\mathbf{b}_{\perp}, \mathbf{p}_{\perp}, x\right)= & \frac{1}{M^{2}} \epsilon_{\perp}^{i j} p_{\perp}^{i} \\
& \times \frac{\partial}{\partial b_{\perp}^{j}} \mathcal{G}_{1,1}^{q}\left(x, 0, \mathbf{p}_{\perp}^{2}, \mathbf{p}_{\perp} \cdot \mathbf{b}_{\perp}, \mathbf{b}_{\perp}^{2}\right), \\
\rho_{L L}^{q}\left(\mathbf{b}_{\perp}, \mathbf{p}_{\perp}, x\right)= & \mathcal{G}_{1,4}^{q}\left(x, 0, \mathbf{p}_{\perp}^{2}, \mathbf{p}_{\perp} \cdot \mathbf{b}_{\perp}, \mathbf{b}_{\perp}^{2}\right),
\end{aligned}
$$

where the $\chi^{q}=\mathcal{F}_{1,1}^{q}, \mathcal{F}_{1,4}^{q}, \mathcal{G}_{1,1}^{q}, \mathcal{G}_{1,4}^{q}$ can be expressed as the Fourier transform of the corresponding GTMDs $X^{q}=$ $F_{1,1}^{q}, F_{1,4}^{q}, G_{1,1}^{q}, G_{1,4}^{q}$,

$$
\begin{aligned}
\chi^{q}\left(x, 0, \mathbf{p}_{\perp}^{2}, \mathbf{p}_{\perp} . \mathbf{b}_{\perp}, \mathbf{b}_{\perp}^{2}\right)= & \int \frac{\mathrm{d}^{2} \Delta_{\perp}}{(2 \pi)^{2}} e^{-i \Delta_{\perp} \cdot \mathbf{b}_{\perp}} \\
& \times X^{q}\left(x, 0, \mathbf{p}_{\perp}^{2}, \mathbf{p}_{\perp} . \Delta_{\perp}, \Delta_{\perp}^{2}\right) .
\end{aligned}
$$

Integrating over all the variables, the Wigner distributions give

$$
\begin{aligned}
& \int \mathrm{d} x \mathrm{~d}^{2} \mathbf{p}_{\perp} \mathrm{d}^{2} \mathbf{b}_{\perp} \rho_{U U}^{q}\left(\mathbf{b}_{\perp}, \mathbf{p}_{\perp}, x\right)=n_{q}, \\
& \int \mathrm{d} x \mathrm{~d}^{2} \mathbf{p}_{\perp} \mathrm{d}^{2} \mathbf{b}_{\perp} \rho_{L U}^{q}\left(\mathbf{b}_{\perp}, \mathbf{p}_{\perp}, x\right)=0, \\
& \int \mathrm{d} x \mathrm{~d}^{2} \mathbf{p}_{\perp} \mathrm{d}^{2} \mathbf{b}_{\perp} \rho_{U L}^{q}\left(\mathbf{b}_{\perp}, \mathbf{p}_{\perp}, x\right)=0, \\
& \int \mathrm{d} x \mathrm{~d}^{2} \mathbf{p}_{\perp} \mathrm{d}^{2} \mathbf{b}_{\perp} \rho_{L L}^{q}\left(\mathbf{b}_{\perp}, \mathbf{p}_{\perp}, x\right)=\Delta q,
\end{aligned}
$$

where the $n_{q}$ is the flavor factors, $n_{u}=2, n_{d}=1$, and $\Delta q$ is the axial charge.

The Wigner distributions cannot have a direct probabilistic interpretation, however, integrating over momentum and position, the Wigner distributions can be reduced to probability distributions. Integrating over $\mathbf{b}_{\perp}$ with $\Delta_{\perp}=0$, the Wigner distributions reduce to the transverse momentum dependent parton distributions (TMDs). At $z_{\perp}=0$, the $\mathbf{p}_{\perp}$ integration of the Wigner distributions give generalized parton distributions (GPDs). The unpolarized TMD $f_{1}^{q}\left(x, \mathbf{p}_{\perp}^{2}\right)$ and GPD $H^{q}\left(x, 0, \Delta_{\perp}^{2}\right)$ can be extracted as

$f_{1}^{q}\left(x, \mathbf{p}_{\perp}^{2}\right)=F_{1,1}^{q}\left(x, 0, \mathbf{p}_{\perp}^{2}, 0,0\right)$,

$H^{q}\left(x, 0, \Delta_{\perp}^{2}\right)=\int \mathrm{d}^{2} \mathbf{p}_{\perp} F_{1,1}^{q}\left(x, 0, \mathbf{p}_{\perp}^{2}, \mathbf{p}_{\perp} . \Delta_{\perp}, \Delta_{\perp}^{2}\right)$,

and the TMD $g_{1 L}^{q}\left(x, \mathbf{p}_{\perp}^{2}\right)$ and GPD $\tilde{H}^{q}\left(x, 0, \Delta_{\perp}^{2}\right)$ can be expressed as 
$g_{1 L}^{q}\left(x, \mathbf{p}_{\perp}^{2}\right)=G_{1,4}^{q}\left(x, 0, \mathbf{p}_{\perp}^{2}, 0,0\right)$,

$\tilde{H}^{q}\left(x, 0, \Delta_{\perp}^{2}\right)=\int \mathrm{d}^{2} \mathbf{p}_{\perp} G_{1,4}^{q}\left(x, 0, \mathbf{p}_{\perp}^{2}, \mathbf{p}_{\perp} . \Delta_{\perp}, \Delta_{\perp}^{2}\right)$.

The $\mathbf{p}_{\perp}$ and $\mathbf{b}_{\perp}$ integration of the $\rho_{L U}^{q}$ and $\rho_{U L}^{q}$ give zero. So, there are no TMD and GPD corresponding to $F_{1,4}$ and $G_{1,1}$ GTMDs.

The Wigner distributions can also be reduced to threedimensional quark densities by integrating over two mutually orthogonal components of transverse position and momentum, e.g. $b_{y}$ and $p_{x}\left(b_{x}\right.$ and $\left.p_{y}\right)$, which are not constrained by the Heisenberg uncertainty principle:

$$
\int \mathrm{d} b_{y} \mathrm{~d} p_{x} \rho^{q[\Gamma]}\left(\mathbf{b}_{\perp}, \mathbf{p}_{\perp}, x ; S\right)=\tilde{\rho}^{q[\Gamma]}\left(b_{x}, p_{y}, x ; S\right),
$$

with $\Delta_{y}=z_{x}=0$. Note that the integration over other mixed transverse components $b_{x}$ and $p_{y}$ gives the same quark density as Eq. (28), with an opposite momentum i.e., $\tilde{\rho}^{q[\Gamma]}\left(b_{y}, p_{x}, x ; S\right)=\tilde{\rho}^{q[\Gamma]}\left(b_{x},-p_{y}, x ; S\right)$. These relations are true only when there is axial symmetry, i.e., for an unpolarized or a longitudinally polarized proton.

\section{Orbital angular momentum}

Jaffe and Manohar showed in the light-cone gauge that the spin of the nucleon can be decomposed into the quark spin, quark OAM, gluon spin, and gluon OAM [35],

$S^{q}+\ell^{q}+S^{g}+\ell^{g}=\frac{1}{2}$.

For the diquark model, the above sum rule can be written as

$S^{q}+\ell^{q}+S^{D}+\ell^{D}=\frac{1}{2}$,

where the super-script $D$ is for the diquark, and for the scalar diquark $S^{D}=0$. The canonical OAM operator for quark is defined as

$\hat{\ell}_{z}^{q}\left(b^{-}, \mathbf{b}_{\perp}, p^{+}, \mathbf{p}_{\perp}\right)=\frac{1}{2} \int \frac{\mathrm{d} z^{-} \mathrm{d}^{2} \mathbf{z}_{\perp}}{(2 \pi)^{3}} e^{i p . z}$

$\bar{\psi}^{q}\left(b^{-}-\frac{z^{-}}{2}, \mathbf{b}_{\perp}\right) \gamma^{+}\left(\mathbf{b}_{\perp} \times(-i \stackrel{\leftrightarrow}{\partial} \perp)\right) \psi^{q}\left(b^{-}+\frac{z^{-}}{2}, \mathbf{b}_{\perp}\right)$.

From the definition of the Wigner operator (Eq. (5)), the OAM density operator can be expressed as

$\hat{\ell}_{z}^{q}=2\left(\mathbf{b}_{\perp} \times \mathbf{p}_{\perp}\right) \hat{W}^{q\left[\gamma^{+}\right]}$.

Thus in the light-front gauge the average canonical OAM for a quark is written in terms of Wigner distribution as

$$
\begin{aligned}
\ell_{z}^{q} & =\int \frac{\mathrm{d} \Delta^{+} \mathrm{d}^{2} \Delta_{\perp}}{2 P^{+}(2 \pi)^{3}}\left\langle P^{\prime \prime} ; S\left|\hat{\ell}_{z}^{q}\right| P^{\prime} ; S\right\rangle \\
& =\int \mathrm{d} x \mathrm{~d}^{2} \mathbf{p}_{\perp} \mathrm{d}^{2} \mathbf{b}_{\perp}\left(\mathbf{b}_{\perp} \times \mathbf{p}_{\perp}\right)_{z} \rho^{q\left[\gamma^{+}\right]}\left(\mathbf{b}_{\perp}, \mathbf{p}_{\perp}, x, \hat{S}_{z}\right) .
\end{aligned}
$$

Here the distribution $\rho^{q\left[\gamma^{+}\right]}\left(\mathbf{b}_{\perp}, \mathbf{p}_{\perp}, x, \hat{S}_{z}\right)$ can be written from Eqs. $(11,12)$ as

$$
\begin{aligned}
\rho^{q\left[\gamma^{+}\right]}\left(\mathbf{b}_{\perp}, \mathbf{p}_{\perp}, x,+\hat{S}_{z}\right)= & \rho_{U U}^{q}\left(\mathbf{b}_{\perp}, \mathbf{p}_{\perp}, x\right) \\
& +\rho_{L U}^{q}\left(\mathbf{b}_{\perp}, \mathbf{p}_{\perp}, x\right) .
\end{aligned}
$$

From Eq. (15) we see that

$\int \mathrm{d} x \mathrm{~d}^{2} \mathbf{p}_{\perp} \mathrm{d}^{2} \mathbf{b}_{\perp}\left(\mathbf{b}_{\perp} \times \mathbf{p}_{\perp}\right)_{z} \rho_{U U}^{q}\left(\mathbf{b}_{\perp}, \mathbf{p}_{\perp}, x\right)=0$,

which satisfies the angular momentum sum rule for an unpolarized proton, the total angular momentum of constituents sum up to zero. Using Eqs. (16) and (19), the twist-2 canonical quark OAM in the light-front gauge is

$\ell_{z}^{q}=-\int \mathrm{d} x \mathrm{~d}^{2} \mathbf{p}_{\perp} \frac{\mathbf{p}_{\perp}^{2}}{M^{2}} F_{1,4}^{q}\left(x, 0, \mathbf{p}_{\perp}^{2}, 0,0\right)$.

The Jaffe-Manohar decomposition (Eq. (29)) is not gauge invariant. Ji proposed a gauge invariant decomposition of the nucleon spin as [36]

$S^{q}+L^{q}+J^{g}=\frac{1}{2}$,

where $L^{q}$ is the kinetic OAM for the quark $q$. However, Chen et al. [37] proposed an idea to decompose the gauge field $A_{\mu}$ into a pure gauge part, $A_{\mu}^{\text {pure }}$, and a physical part, $A_{\mu}^{\text {phy }}$ to give a gauge invariant definition of the Jaffe-Manohar decomposition.

The kinetic OAM of quark appearing in the Ji sum rule is defined in terms of GPDs as [36]

$$
\begin{aligned}
L_{z}^{q}= & \frac{1}{2} \int \mathrm{d} x\left[x\left(H^{q}(x, 0,0)+E^{q}(x, 0,0)\right)\right. \\
& \left.-\tilde{H}^{q}(x, 0,0)\right]
\end{aligned}
$$

where $H^{q}(x, \xi, t)$ and $E^{q}(x, \xi, t)$ are unpolarized GPDs and $\tilde{H}^{q}(x, \xi, t)$ is the helicity dependent GPD. In our model calculation, the explicit expressions are given in Sect. 5. A comparative study between the longitudinal component of the canonical OAM and the kinetic OAM is shown in Fig. 1 and the values are given in Table 2. Note that the above relation (Eq. 38) does not hold for the density level interpretation in the transverse plane [38].

The spin-orbit correlation is given by the operator

$$
\begin{aligned}
& C_{z}^{q}\left(b^{-}, \mathbf{b}_{\perp}, p^{+}, \mathbf{p}_{\perp}\right)=\frac{1}{2} \int \frac{\mathrm{d} z^{-} \mathrm{d}^{2} \mathbf{z}_{\perp}}{(2 \pi)^{3}} e^{i p . z} \\
& \bar{\psi}^{q}\left(b^{-}-\frac{z^{-}}{2}, \mathbf{b}_{\perp}\right) \gamma^{+} \gamma^{5}\left(\mathbf{b}_{\perp} \times(-i \stackrel{\leftrightarrow}{\partial} \perp)\right) \psi^{q}\left(b^{-}+\frac{z^{-}}{2}, \mathbf{b}_{\perp}\right) .
\end{aligned}
$$



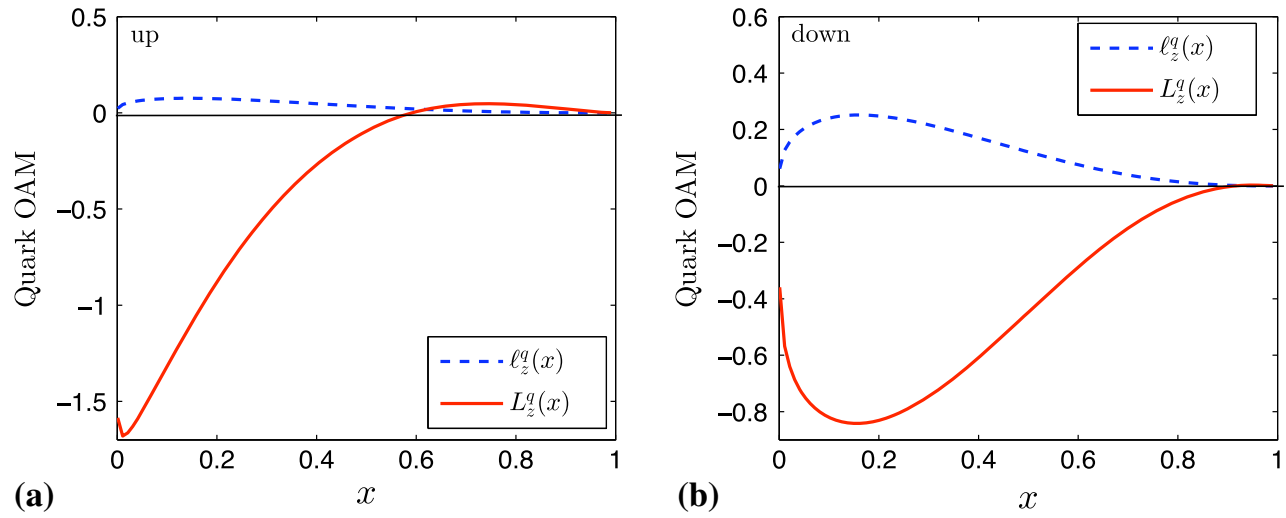

Fig. 1 The variation of canonical OAM $\ell_{z}^{q}(x)$ and kinetic OAM $L_{z}^{q}(x)$ with longitudinal momentum fraction $x$, for a $u$ quark and $\mathbf{b} d$ quark

Table 2 In the light-front AdS/QCD scalar diquark model, the values of canonical OAM $\ell_{z}^{q}$ and the kinetic OAM $L_{z}^{q}$ for the $u$ and the $d$ quark

\begin{tabular}{lccr}
\hline & $q$-OAM & $u$ & \multicolumn{1}{c}{$d$} \\
\hline Canonical & $\ell_{z}^{q}$ & 0.0348 & 0.1201 \\
Kinetic & $L_{z}^{q}$ & -0.3812 & -0.4258 \\
\hline
\end{tabular}

The correlation between quark spin and quark OAM can be expressed with Wigner distributions $\rho_{U L}^{q}$ and equivalently in terms of GTMD as

$$
\begin{aligned}
C_{z}^{q} & =\int \mathrm{d} x \mathrm{~d}^{2} \mathbf{p}_{\perp} \mathrm{d}^{2} \mathbf{b}_{\perp}\left(\mathbf{b}_{\perp} \times \mathbf{p}_{\perp}\right)_{z} \rho_{U L}^{q}\left(\mathbf{b}_{\perp}, \mathbf{p}_{\perp}, x\right) \\
& =\int \mathrm{d} x \mathrm{~d}^{2} \mathbf{p}_{\perp} \frac{\mathbf{p}_{\perp}^{2}}{M^{2}} G_{1,1}^{q}\left(x, 0, \mathbf{p}_{\perp}^{2}, 0,0\right),
\end{aligned}
$$

where $C_{z}^{q}>0$ implies the quark spin and OAM tend to be aligned and $C_{z}^{q}<0$ implies they are anti-aligned. In our model, the quark spin and OAM tend to be anti-aligned for both $u$ and $d$ quarks.

One can see from Eq. (18) that a similar correlator with $\rho_{L L}^{q}$ vanishes,

$$
\int \mathrm{d} x \mathrm{~d}^{2} \mathbf{p}_{\perp} \mathrm{d}^{2} \mathbf{b}_{\perp}\left(\mathbf{b}_{\perp} \times \mathbf{p}_{\perp}\right)_{z} \rho_{L L}^{q}\left(\mathbf{b}_{\perp}, \mathbf{p}_{\perp}, x\right)=0 .
$$

\section{Results}

We calculate the Wigner distributions of the proton in the light-front AdS/QCD quark-diquark model. Using Eq. (1) in Eq. (5) the quark-quark correlator, $W^{q[\Gamma]}\left(\Delta_{\perp}, \mathbf{p}_{\perp}, x ; S\right)$, can be expressed in terms of LFWFs as

$$
W^{q\left[\gamma^{+}\right]}\left(\Delta_{\perp}, \mathbf{p}_{\perp}, x ; \pm \hat{S}_{z}\right)=\frac{1}{16 \pi^{3}}\left[\psi_{q+}^{ \pm \dagger}\left(x, \mathbf{p}_{\perp}^{\prime \prime}\right) \psi_{q+}^{ \pm}\left(x, \mathbf{p}_{\perp}^{\prime}\right)\right.
$$

$$
\begin{aligned}
& \left.+\psi_{q-}^{ \pm \dagger}\left(x, \mathbf{p}_{\perp}^{\prime \prime}\right) \psi_{q-}^{ \pm}\left(x, \mathbf{p}_{\perp}^{\prime}\right)\right], \\
& W^{q\left[\gamma^{+} \gamma^{5}\right]}\left(\Delta_{\perp}, \mathbf{p}_{\perp}, x ; \pm \hat{S}_{z}\right)=\frac{1}{16 \pi^{3}}\left[\psi_{q+}^{ \pm \dagger}\left(x, \mathbf{p}_{\perp}^{\prime \prime}\right) \psi_{q+}^{ \pm}\left(x, \mathbf{p}_{\perp}^{\prime}\right)\right. \\
& \left.-\psi_{q-}^{ \pm \dagger}\left(x, \mathbf{p}_{\perp}^{\prime \prime}\right) \psi_{q-}^{ \pm}\left(x, \mathbf{p}_{\perp}^{\prime}\right)\right]
\end{aligned}
$$

for the Dirac structures $\Gamma=\gamma^{+}, \gamma^{+} \gamma^{5}$. In the symmetric frame the initial and final momenta of the struck quark are

$$
\begin{aligned}
& \mathbf{p}_{\perp}^{\prime}=\mathbf{p}_{\perp}-(1-x) \frac{\Delta_{\perp}}{2}, \\
& \mathbf{p}_{\perp}^{\prime \prime}=\mathbf{p}_{\perp}+(1-x) \frac{\Delta_{\perp}}{2},
\end{aligned}
$$

respectively. Using the wave functions from Eqs. $(2,3)$ in Eqs. (42, 43), the explicit expressions for the Wigner distributions are

$$
\begin{aligned}
& \rho_{U U}^{q}\left(\mathbf{b}_{\perp}, \mathbf{p}_{\perp}, x\right)=\frac{1}{16 \pi^{3}} \int \frac{\mathrm{d} \Delta_{\perp}}{2 \pi} \Delta_{\perp} \mathrm{J}_{0}\left(\left|\Delta_{\perp}\right|\left|b_{\perp}\right|\right) \\
& \quad \times \exp \left(-2 \tilde{a}(x) \tilde{\mathbf{p}}_{\perp}^{2}\right)\left[\left|A_{q}^{(1)}(x)\right|^{2}\right. \\
& \left.\quad+\left(\mathbf{p}_{\perp}^{2}-\frac{\Delta_{\perp}^{2}}{4}(1-x)^{2}\right) \frac{1}{M^{2} x^{2}}\left|A_{q}^{(2)}(x)\right|^{2}\right], \\
& \rho_{L U}^{q}\left(\mathbf{b}_{\perp}, \mathbf{p}_{\perp}, x\right)=-\frac{1}{M^{2}} \epsilon_{\perp}^{i j} p_{\perp}^{i} \frac{\partial}{\partial b_{\perp}^{j}}\left[-\frac{1}{16 \pi^{3}} \int \frac{\mathrm{d} \Delta_{\perp}}{2 \pi}\right. \\
& \left.\quad \times \Delta_{\perp} \mathrm{J}_{0}\left(\left|\Delta_{\perp}\right|\left|b_{\perp}\right|\right) \exp \left(-2 \tilde{a}(x) \tilde{\mathbf{p}}_{\perp}^{2}\right) \frac{(1-x)}{x^{2}}\left|A_{q}^{(2)}(x)\right|^{2}\right],
\end{aligned}
$$

$$
\begin{aligned}
& \rho_{U L}^{q}\left(\mathbf{b}_{\perp}, \mathbf{p}_{\perp}, x\right)=\frac{1}{M^{2}} \epsilon_{\perp}^{i j} p_{\perp}^{i} \frac{\partial}{\partial b_{\perp}^{j}}\left[-\frac{1}{16 \pi^{3}} \int \frac{\mathrm{d} \Delta_{\perp}}{2 \pi}\right. \\
& \left.\quad \times \Delta_{\perp} \mathrm{J}_{0}\left(\left|\Delta_{\perp}\right|\left|b_{\perp}\right|\right) \exp \left(-2 \tilde{a}(x) \tilde{\mathbf{p}}_{\perp}^{2}\right) \frac{(1-x)}{x^{2}}\left|A_{q}^{(2)}(x)\right|^{2}\right],
\end{aligned}
$$

$\rho_{L L}^{q}\left(\mathbf{b}_{\perp}, \mathbf{p}_{\perp}, x\right)=\frac{1}{16 \pi^{3}} \int \frac{\mathrm{d} \Delta_{\perp}}{2 \pi} \Delta_{\perp} \mathrm{J}_{0}\left(\left|\Delta_{\perp}\right|\left|b_{\perp}\right|\right)$ 


$$
\begin{aligned}
& \times \exp \left(-2 \tilde{a}(x) \tilde{\mathbf{p}}_{\perp}^{2}\right)\left[\left|A_{q}^{(1)}(x)\right|^{2}\right. \\
& \left.-\left(\mathbf{p}_{\perp}^{2}-\frac{\Delta_{\perp}^{2}}{4}(1-x)^{2}\right) \frac{1}{M^{2} x^{2}}\left|A_{q}^{(2)}(x)\right|^{2}\right],
\end{aligned}
$$

where

$$
\begin{aligned}
A_{q}^{(i)}(x) & =N_{q}^{(i)} \frac{4 \pi}{\kappa} \sqrt{\frac{\log (1 / x)}{(1-x)}} x_{q}^{a_{q}^{(i)}}(1-x)^{b_{q}^{(i)}}, \\
\tilde{a}(x) & =\frac{\log (1 / x)}{2 \kappa^{2}(1-x)^{2}}, \\
\tilde{\mathbf{p}}_{\perp}^{2} & =\mathbf{p}_{\perp}^{2}+\frac{\Delta_{\perp}^{2}}{4}(1-x)^{2} .
\end{aligned}
$$

In the limit $\xi=0$, the GTMDs are

$$
\begin{aligned}
& F_{1,1}^{q}\left(x, \Delta_{\perp}^{2}, \mathbf{p}_{\perp}^{2}\right)=\frac{1}{16 \pi^{3}}\left[\left|A_{q}^{(1)}(x)\right|^{2}+\left(\mathbf{p}_{\perp}^{2}-\frac{\Delta_{\perp}^{2}}{4}(1-x)^{2}\right)\right. \\
& \left.\quad \times \frac{1}{M^{2} x^{2}}\left|A_{q}^{(2)}(x)\right|^{2}\right] \exp \left[-2 \tilde{a}(x) \tilde{\mathbf{p}}_{\perp}^{2}\right] \\
& F_{1,4}^{q}\left(x, \Delta_{\perp}, \mathbf{p}_{\perp}^{2}\right)=-\frac{1}{16 \pi^{3}}\left[\frac{(1-x)}{x^{2}}\left|A_{q}^{(2)}(x)\right|^{2}\right] \\
& \quad \times \exp \left[-2 \tilde{a}(x) \tilde{\mathbf{p}}_{\perp}^{2}\right], \\
& G_{1,1}^{q}\left(x, \Delta_{\perp}^{2}, \mathbf{p}_{\perp}^{2}\right)=-\frac{1}{16 \pi^{3}}\left[\frac{(1-x)}{x^{2}}\left|A_{q}^{(2)}(x)\right|^{2}\right] \\
& \quad \times \exp \left[-2 \tilde{a}(x) \tilde{\mathbf{p}}_{\perp}^{2}\right] \\
& G_{1,4}^{q}\left(x, \Delta_{\perp}, \mathbf{p}_{\perp}^{2}\right)=\frac{1}{16 \pi^{3}}\left[\left|A_{q}^{(1)}(x)\right|^{2}-\left(\mathbf{p}_{\perp}^{2}-\frac{\Delta_{\perp}^{2}}{4}(1-x)^{2}\right)\right. \\
& \left.\quad \times \frac{1}{M^{2} x^{2}}\left|A_{q}^{(2)}(x)\right|^{2}\right] \exp \left[-2 \tilde{a}(x) \tilde{\mathbf{p}}_{\perp}^{2}\right]
\end{aligned}
$$

We find $F_{1,4}^{q}=G_{1,1}^{q}$ to the leading order as found in [39] for the scalar diquark model. Thus the distributions $\rho_{L U}^{q}=$ $-\rho_{U L}^{q}$.

From Eq. (36), the canonical OAM can be written as

$\ell_{z}^{q}=\int \mathrm{d} x \ell_{z}^{q}(x)$.

Using Eq. (54), $\ell_{z}^{q}(x)$ can be written as

$\ell_{z}^{q}(x)=\frac{\kappa^{2}}{M^{2} \log (1 / x)} F_{2}^{q}(x)(1-x)^{5}$.

In this model, $\ell_{z}^{q}$ can also be related with the pretzelosity $h_{1 T}^{\perp}$ as

$\ell_{z}^{q}=-\int \mathrm{d} x \mathrm{~d}^{2} \mathbf{p}_{\perp} \frac{\mathbf{p}_{\perp}^{2}}{2 M^{2}}(1-x) h_{1 T}^{q \perp}\left(x, \mathbf{p}_{\perp}^{2}\right)$.

$h_{1 T}^{q \perp}\left(x, \mathbf{p}_{\perp}^{2}\right)$ is one of the eight leading twist TMDs. In this light-front scalar diquark model $h_{1 T}^{q \perp}\left(x, \mathbf{p}_{\perp}^{2}\right)$ is written as [30]

$h_{1 T}^{q \perp}\left(x, \mathbf{p}_{\perp}^{2}\right)=-\frac{2 \log (1 / x)}{\pi \kappa^{2}} \exp \left[-\frac{\mathbf{p}_{\perp}^{2} \log (1 / x)}{\kappa^{2}(1-x)^{2}}\right] F_{2}^{q}(x)$, where $F_{2}^{q}(x)$ is given in Eq. (67). Using Eq. (53) and Eq. (56) in Eqs. (25) and (27), the GPDs $H$ and $\tilde{H}$ can be expressed as

$$
\begin{aligned}
H^{q}(x, 0, t)= & {\left[F_{1}^{q}(x)(1-x)^{2}+F_{2}^{q}(x)(1-x)^{4} \frac{\kappa^{2}}{M^{2} \log (1 / x)}\right] } \\
& \times\left(1-\frac{|t|}{4 \kappa^{2}} \log (1 / x)\right) \exp \left[-\frac{|t|}{4 \kappa^{2}} \log (1 / x)\right], \\
\tilde{H}^{q}(x, 0, t)= & {\left[F_{1}^{q}(x)(1-x)^{2}-F_{2}^{q}(x)(1-x)^{4} \frac{\kappa^{2}}{M^{2} \log (1 / x)}\right] } \\
& \times\left(1-\frac{|t|}{4 \kappa^{2}} \log (1 / x)\right) \exp \left[-\frac{|t|}{4 \kappa^{2}} \log (1 / x)\right] .
\end{aligned}
$$

In the AdS/QCD light-front scalar diquark model the helicity flip GPD $E$ is given [27] as

$E^{q}(x, 0, t)=2 F_{3}^{q}(x)(1-x)^{3} \exp \left[-\frac{|t|}{4 \kappa^{2}} \log (1 / x)\right]$,

where $Q^{2}=-q^{2}=-t$, the square of the momentum transferred in the process, and it is taken to be zero for the OAM calculation.

The kinetic OAM of quarks (Eq. (38)) can be written as

$L_{z}^{q}=\int \mathrm{d} x L_{z}^{q}(x)$.

In this model, using Eqs. (61), (62), and (63) in the $t=0$ limit, the $L_{z}^{q}(x)$ reads

$$
\begin{aligned}
L_{z}^{q}(x)= & \frac{1}{2}\left[-F_{1}^{q}(x)(1-x)^{3}+F_{2}^{q}(x)(1-x)^{4}(1+x)\right. \\
& \left.\times \frac{\kappa^{2}}{M^{2} \log (1 / x)}+2 F_{3}^{q}(x) x(1-x)^{3}\right],
\end{aligned}
$$

where

$$
\begin{aligned}
& F_{1}^{q}(x)=\left|N_{q}^{(1)}\right|^{2} x^{2 a_{q}^{(1)}}(1-x)^{2 b_{q}^{(1)}-1}, \\
& F_{2}^{q}(x)=\left|N_{q}^{(2)}\right|^{2} x^{2 a_{q}^{(2)}-2}(1-x)^{2 b_{q}^{(2)}-1}, \\
& F_{3}^{q}(x)=N_{q}^{(1)} N_{q}^{(2)} x^{a^{(1)}+a_{q}^{(2)}-1}(1-x)^{b_{q}^{(1)}+b_{q}^{(2)}-1} .
\end{aligned}
$$

The variation of the quark OAMs $\ell_{z}^{q}(x)$ and $L_{z}^{q}(x)$ with longitudinal momentum fraction $x$ is shown in Fig. 1 for the $u$ and the $d$ quark.

\subsection{Unpolarized proton}

In our numerical study, we have considered the active quark to be either a $u$ or $d$ quark, the spectator always being a diquark. In other words, when we calculate the Wigner distribution for the $u$ quark, we have not incorporated any contribution from the $u$ quark that is part of the diquark. The first Mellin moment of $\rho_{U U}^{q}\left(\mathbf{b}_{\perp}, \mathbf{p}_{\perp}, x\right)$ is shown in Fig. 2. Figure 2a, $\mathrm{b}$ represent the distributions in transverse momentum plane 

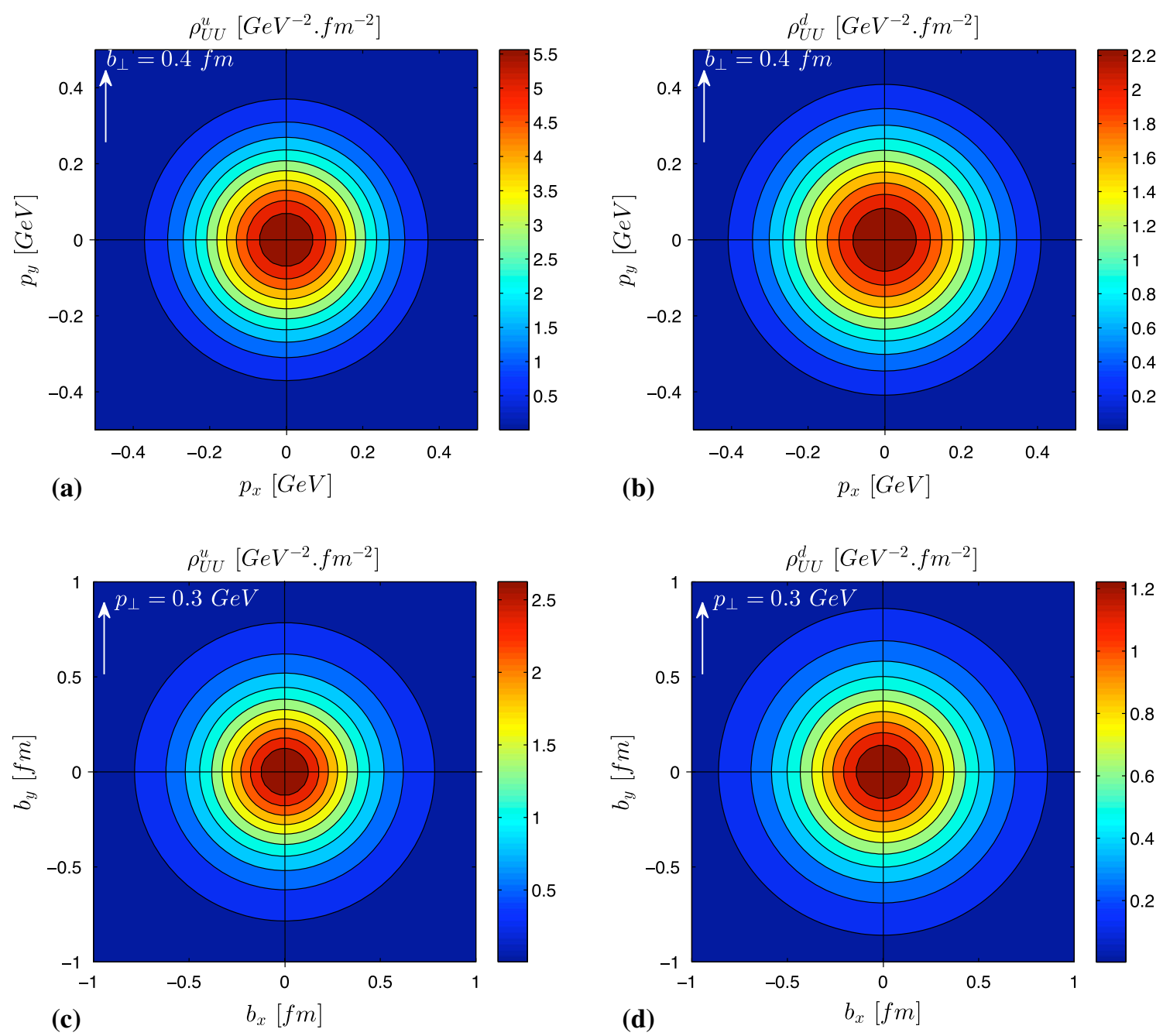

Fig. 2 The Wigner distributions of unpolarized quarks in an unpolarized proton in the transverse momentum plane $(\mathbf{a}, \mathbf{b})$ with $\mathbf{b}_{\perp}=0.4 \hat{y} f m$ and in the transverse impact parameter plane (c, $\mathbf{d})$ with $\mathbf{p}_{\perp}=0.3 \hat{y} \mathrm{GeV}$ for the $u$ quarks (left column) and $d$ quarks (right column)

for the $u$ quark and $d$ quark, respectively. The fixed impact parameter $\mathbf{b}_{\perp}$ is taken along $\hat{y}$ and $b_{y}=0.4 \mathrm{fm}$.

The variation of $\rho_{U U}^{q}\left(\mathbf{b}_{\perp}, \mathbf{p}_{\perp}\right)$ in the transverse impact parameter plane are shown in Fig. 2c, $\mathrm{d}$ for the $u$ and the $d$ quark, respectively, with fixed transverse momentum $\mathbf{p}_{\perp}$ along $\hat{y}$ for $p_{y}=0.3 \mathrm{GeV}$. The distributions $\rho_{U U}^{u}$ and $\rho_{U U}^{d}$ are circularly symmetric, in transverse momentum plane as well as transverse impact parameter plane, with a positive maximum at the center $\left(p_{x}=p_{y}=0\right),\left(b_{x}=b_{y}=0\right)$ and gradually decrease toward the periphery, for both $u$ and $d$ quarks. The peak of the distribution for the $u$ quark is large compared to the $d$ quark in the two planes.

The average quadrupole distortions $Q_{b}^{i j}\left(\mathbf{p}_{\perp}\right)$ and $Q_{p}^{i j}\left(\mathbf{b}_{\perp}\right)$ are defined as [11]

$$
Q_{b}^{i j}\left(\mathbf{p}_{\perp}\right)=\frac{\int \mathrm{d}^{2} \mathbf{b}_{\perp}\left(2 b_{\perp}^{i} b_{\perp}^{j}-\delta^{i j} \mathbf{b}_{\perp}^{2}\right) \rho_{U U}\left(\mathbf{b}_{\perp}, \mathbf{p}_{\perp}\right)}{\int \mathrm{d}^{2} \mathbf{b}_{\perp} \mathbf{b}_{\perp}^{2} \rho_{U U}\left(\mathbf{b}_{\perp}, \mathbf{p}_{\perp}\right)}
$$

$$
Q_{p}^{i j}\left(\mathbf{b}_{\perp}\right)=\frac{\int \mathrm{d}^{2} \mathbf{p}_{\perp}\left(2 p_{\perp}^{i} p_{\perp}^{j}-\delta^{i j} \mathbf{p}_{\perp}^{2}\right) \rho_{U U}\left(\mathbf{b}_{\perp}, \mathbf{p}_{\perp}\right)}{\int \mathrm{d}^{2} \mathbf{p}_{\perp} \mathbf{p}_{\perp}^{2} \rho_{U U}\left(\mathbf{b}_{\perp}, \mathbf{p}_{\perp}\right)} .
$$

In this model, the average quadrupole distortion is found to be zero. Since the wave functions in soft-wall AdS/QCD model are of gaussian type, $\rho_{U U}$ and $\rho_{L L}$ are even in $\mathbf{p}_{\perp}$ and $\mathbf{b}_{\perp}$, resulting in a zero quadrupole distortion.

As we discussed before, the three dimensional quark densities can be extracted from the Wigner distributions by integrating over one transverse momentum $p_{x}$ and one transverse position $b_{y}$ variables (see Eq. (28)). The $\tilde{\rho}_{U U}\left(b_{x}, p_{y}\right)$ in the mixed transverse plane are shown in Fig. 3 for the $u$ and the $d$ quarks. We find that the distributions are axially symmetric. Therefore, there is no favored configuration between $\mathbf{b}_{\perp} \perp \mathbf{p}_{\perp}$ and $\mathbf{b}_{\perp} \| \mathbf{p}_{\perp}$ unlike the lightcone constituent quark model (LCCQM) [12-16] or chiral quark soliton model ( $\chi \mathrm{QSM})[18-20]$. At $b_{x}=p_{y}=0$, the probability density for the $u$ and the $d$ quark is maxi- 

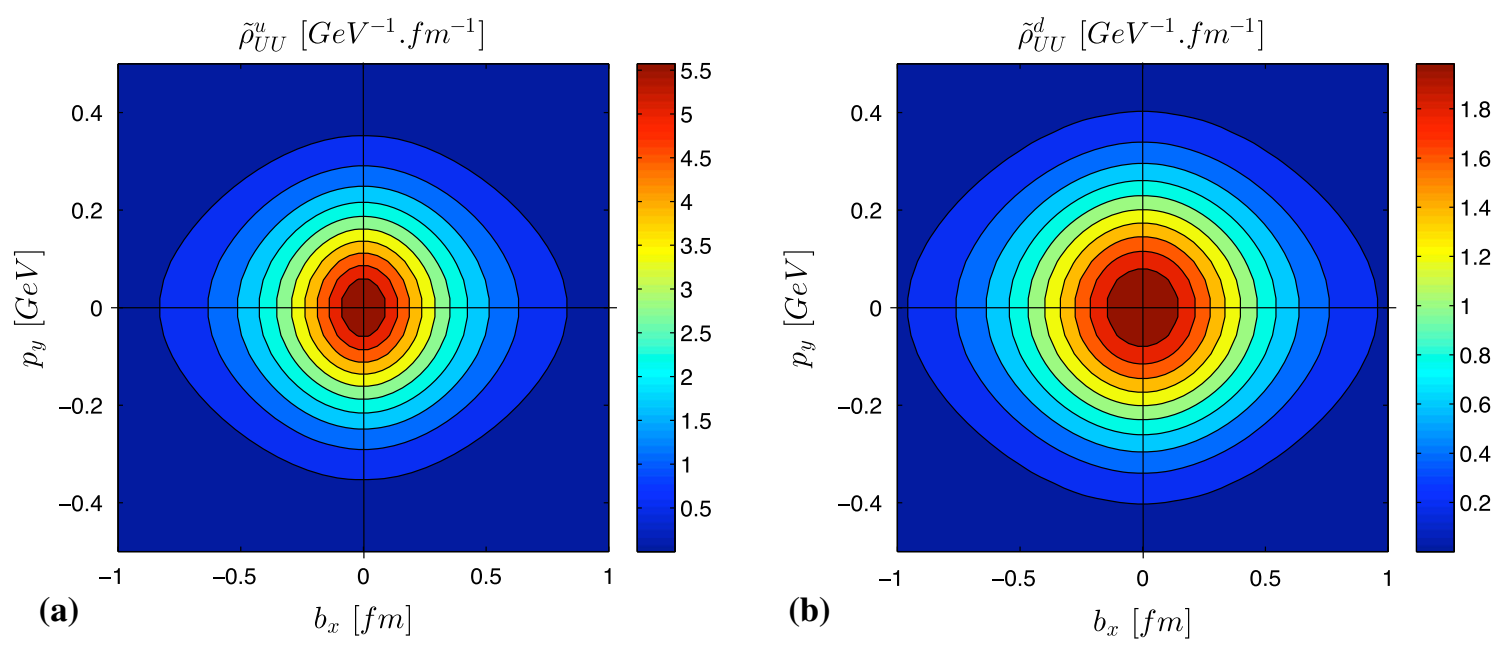

Fig. $3 \tilde{\rho}_{U U}^{q}\left(b_{x}, p_{y}\right)$ in mixed transverse plane for the $u$ quark (a) and the $d$ quark (b)

mum and decreases as $e^{-\alpha p_{y}^{2}}$ and $e^{-\beta b_{x}^{2}}$. Here $\alpha$ and $\beta$ are positive constants and we observe $\alpha>\beta$ for both $u$ and $d$ quarks.

The Wigner distributions $\rho_{U L}^{q}\left(\mathbf{b}_{\perp}, \mathbf{p}_{\perp}\right)$, in the transverse momentum plane, are shown in Fig. $4 \mathrm{a}, \mathrm{b}$ for the $u$ and the $d$ quarks, respectively. The fixed transverse impact parameter $\mathbf{b}_{\perp}$ is along $\hat{y}$ with $b_{y}=0.4 \mathrm{fm}$. Figure $4 \mathrm{c}$, d represent the distribution $\rho_{U L}^{q}\left(\mathbf{b}_{\perp}, \mathbf{p}_{\perp}\right)$ in the transverse impact parameter plane, for the $u$ and the $d$ quark for $\mathbf{p}_{\perp}=p_{y} \hat{y}=0.3 \mathrm{GeV}$. We observe dipolar distributions having the same polarity for the $u$ and the $d$ quarks.

The $\tilde{\rho}_{U L}^{q}\left(b_{x}, p_{y}\right)$ in the transverse mixed plane are shown in Fig. 5. We find a quadrupole distribution for the $u$ and the $d$ quarks. Using Eq. (55) in Eq. (40) we calculate the $C_{z}^{q}$, the correlation between quark spin and quark OAM. The values are: $C_{z}^{u}=-0.0348$ for $u$ quark and $C_{z}^{d}=-0.1201$ for the $d$ quarks. Therefore in this model, the quark OAM tends to be anti-aligned $\left(C_{z}^{u}<0, C_{z}^{d}<0\right)$ to the quark spin for both $u$ and $d$ quarks.

\subsection{Longitudinally polarized proton}

The Wigner distributions $\rho_{L U}^{q}\left(\mathbf{b}_{\perp}, \mathbf{p}_{\perp}\right)$ are shown in Fig. 6 for the $u$ and the $d$ quarks. Figure $6 \mathrm{a}, \mathrm{b}$ show the variation of $\rho_{L U}^{q}\left(\mathbf{b}_{\perp}, \mathbf{p}_{\perp}\right)$ in transverse momentum plane for the $u$ and the $d$ quarks, respectively, with $\mathbf{b}_{\perp}$ along $\hat{y}$ and $b_{y}=0.4 \mathrm{fm}$. The variation of $\rho_{L U}^{q}\left(\mathbf{b}_{\perp}, \mathbf{p}_{\perp}\right)$ in the transverse impact parameter plane is shown in Fig. $6 c$, d with fixed $\mathbf{p}_{\perp}$ along $\hat{y}, p_{y}=0.3 \mathrm{GeV}$. We find dipolar distributions for the $u$ and the $d$ quarks. The polarity of the dipolar distribution $\rho_{L U}^{q}$ is opposite to the polarity of $\rho_{U L}^{q}$. The maximum value of $\rho_{L U}^{q}\left(\mathbf{b}_{\perp}, \mathbf{p}_{\perp}\right)$ for the $u$ quark is less than that for the $d$ quarks in the two planes.

Figure $7 \mathrm{a}, \mathrm{b}$ represent the distribution $\tilde{\rho}_{L U}^{q}\left(b_{x}, p_{y}\right)$ in the mixed transverse plane for the $u$ and the $d$ quarks, respec- tively. We observe quadrupole distributions for both $u$ and $d$ quarks. The quadrupole structures in $\rho_{L U}^{q}\left(\mathbf{b}_{\perp}, \mathbf{p}_{\perp}\right)$ and $\rho_{U L}^{q}\left(\mathbf{b}_{\perp}, \mathbf{p}_{\perp}\right)$ are found due to the presence of the derivative terms in Eqs. (16) and (17).

From Eqs. (57) and (64), we calculate the canonical OAM and kinetic OAM of the quarks in this model. The values of quark OAM are given in Table 2. Note that in quark-diquark model, the total proton OAM is given by the sum of quark and diquark angular momenta, so unlike the quark models the $u$ and $d$ quark contributions do not add up to the total proton OAM and hence the sum of kinetic OAM of $u$ and $d$ in Table 2 is not the same as total canonical OAM of the $u$ and $d$. The correlation between the canonical OAM of quark and proton spin can be understood from the sign of the $\ell_{z}^{q}$. In our model calculation, the positive values of $\ell_{z}^{q}$ for both $u$ and $d$ imply that the proton spin tends to be aligned to quark OAM for both $u$ and $d$ quarks. The spin contribution of the quark to the proton spin is given by [11]

$$
\begin{aligned}
s^{q}=\frac{1}{2} \Delta q & =\frac{1}{2} \int \mathrm{d} x \tilde{H}^{q}(x, 0,0) \\
& =\frac{1}{2} \int \mathrm{d} x \mathrm{~d}^{2} p_{\perp} G_{1,4}^{q}\left(x, 0, \mathbf{p}_{\perp}^{2}, 0,0\right)
\end{aligned}
$$

where $\Delta q$ is the axial charge. In our model, we get $s^{u}=$ 0.946 and $s^{d}=0.396$. It is well known that the spectator diquark model has its own limitations [40]. Though the functional behaviors of the GPDs and GTMDs are well reproduced in our model, the axial charges for both $u$ and $d$ quarks are over estimated. The model is defined at a very low scale $Q_{0}^{2} \approx 0.09 \mathrm{GeV}^{2}$. The axial charge is scale dependent and known to be negative at larger scales. In [26], the authors have extended the result to an arbitrary scale $Q^{2}$ and studied the evolution of unpolarized PDFs in this model. Our result agrees closely with theirs, in spite of the fact that the fit parameters are slightly different. In their model [41], 

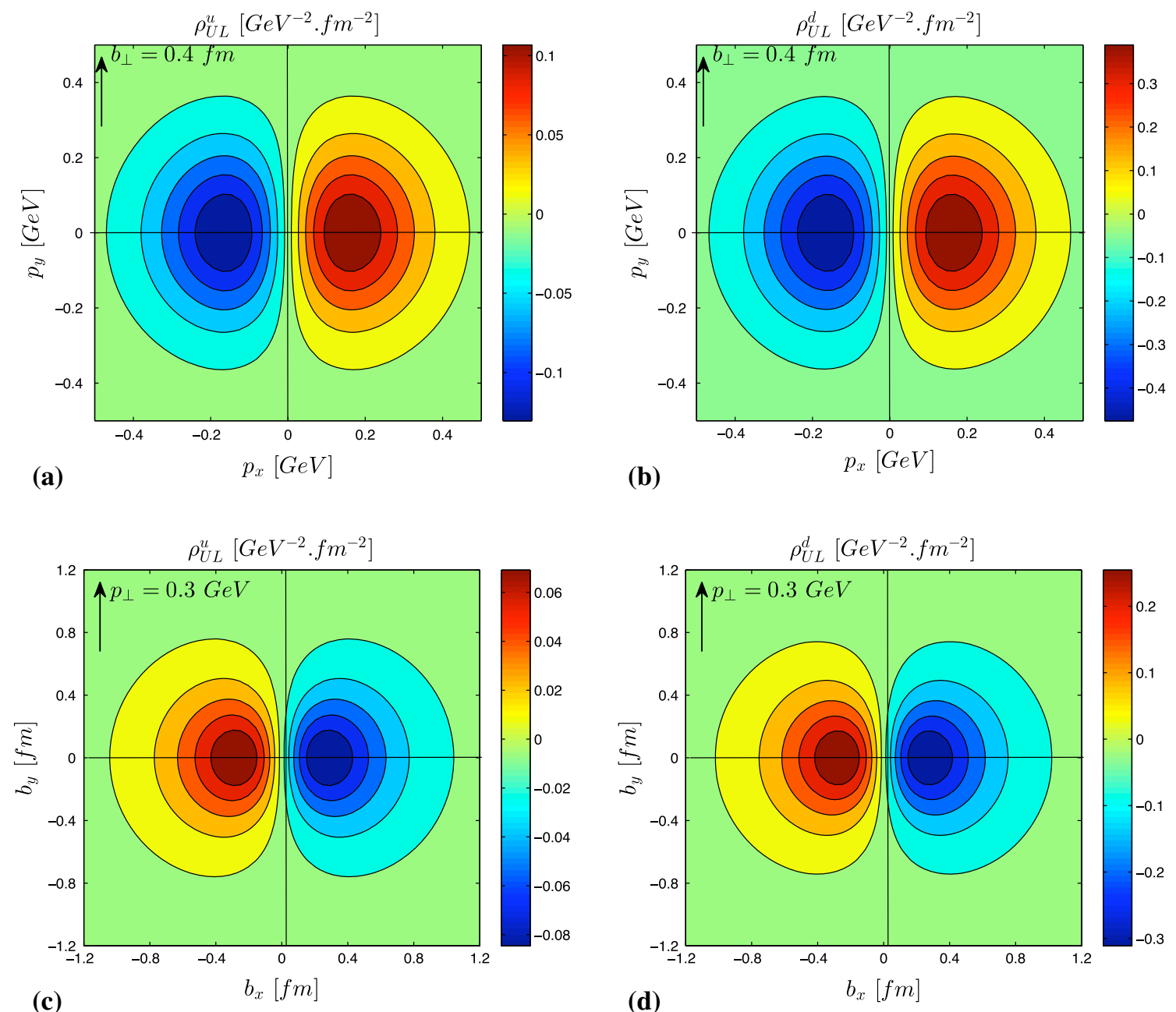

Fig. 4 The dipolar behavior of $\rho_{U L}^{q}$ in the transverse momentum plane $(\mathbf{a}, \mathbf{b})$ with $\mathbf{b}_{\perp}=0.4 \hat{y} f m$ and in the transverse impact parameter plane (c, $\mathbf{d}$ ) with $\mathbf{p}_{\perp}=0.3 \hat{y} \mathrm{GeV}$ for the $u$ quarks (left column) and $d$ quarks (right column)
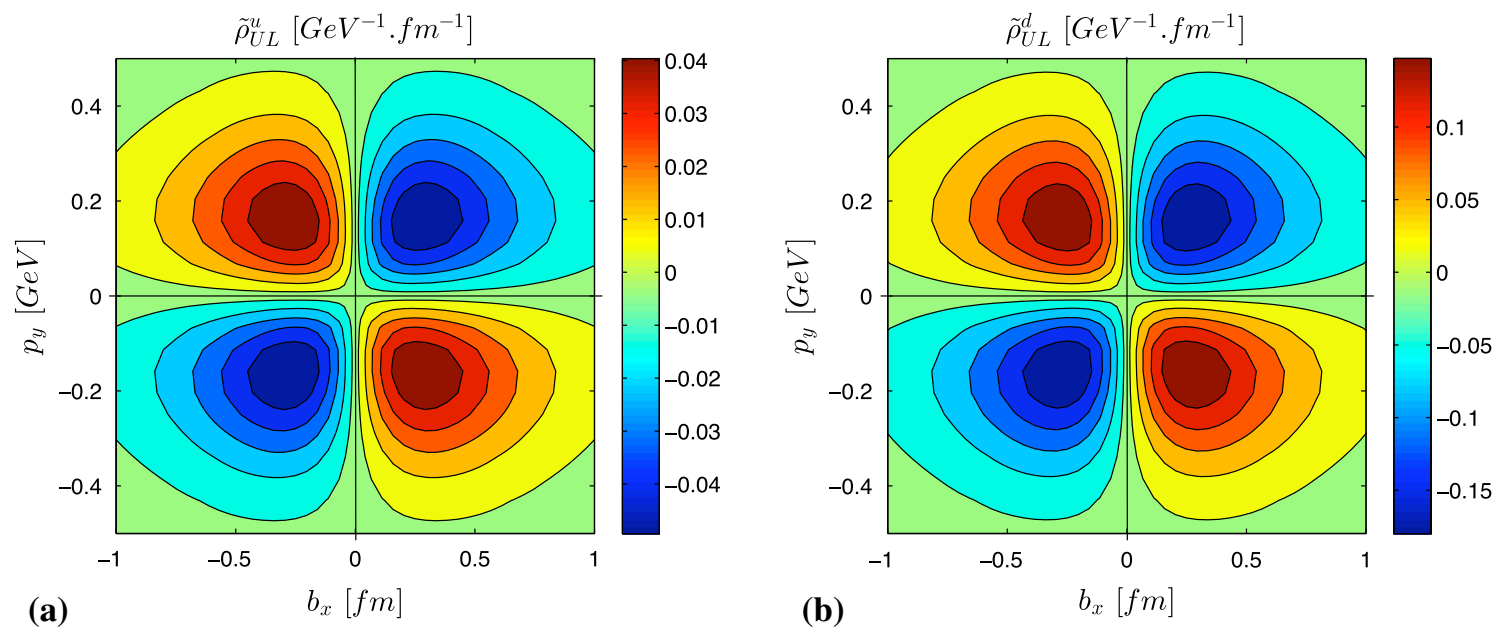

Fig. $5 \tilde{\rho}_{U L}^{q}\left(b_{x}, p_{y}\right)$ in mixed transverse plane corresponding to $u$ quark (a) and $d$ quark (b) 

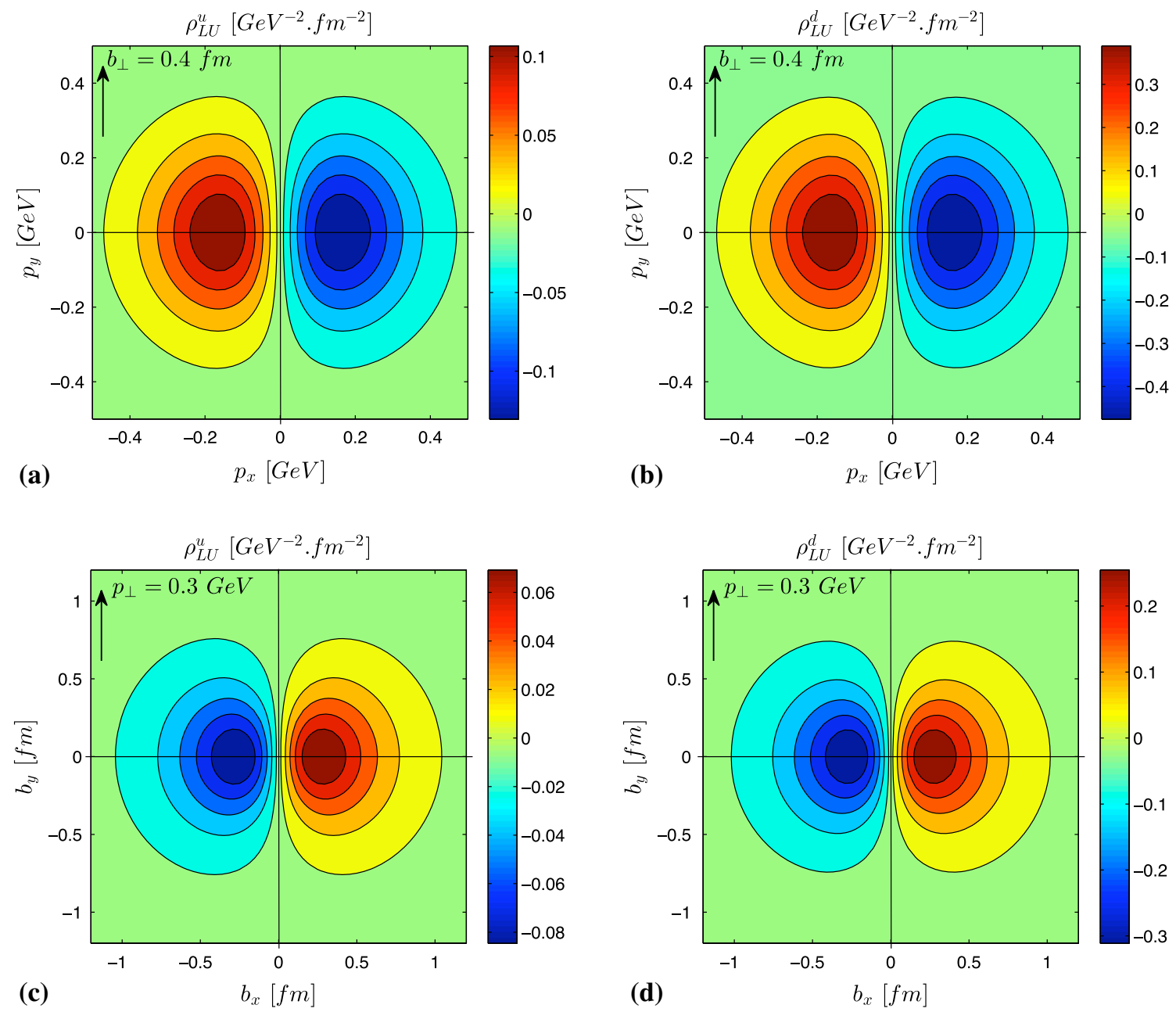

Fig. 6 The dipolar behavior of $\rho_{L U}^{q}$ in the transverse momentum plane $(\mathbf{a}, \mathbf{b})$ with $\mathbf{b}_{\perp}=0.4 \hat{y} \mathrm{fm}$ and in the transverse impact parameter plane (c, $\mathbf{d}$ ) with $\mathbf{p}_{\perp}=0.3 \hat{y} \mathrm{GeV}$ for the $u$ quarks (left column) and $d$ quarks (right column)

the PDFs are slightly smaller in magnitude. When polarized PDFs or helicity distributions are computed, the $d$ quark helicity distribution turns out to be positive, although it is expected to be negative from the recent fit of the data [42]. In $[43,44]$, it has been shown that NNPDF allows for a positive total $\Delta \mathrm{d}(x) / \mathrm{d}(x)$ (where $\Delta \mathrm{d}(x)$ stands for helicity distribution) for larger values of $x$, this is also obtained in some other models; for example in [45] the above ratio was calculated in perturbative QCD taking into account the valence Fock components with non-vanishing orbital angular momentum and it was found that $\Delta \mathrm{d}(x) / \mathrm{d}(x)$ is positive as $x \approx 0.75$ and approaches 1 as $x \rightarrow 1$. Positive values of this ratio were also found in an SU(6) breaking quark model calculation in [46]. Another way to parametrize the model would be to fit the data of the helicity distributions with the model parameters, instead of the form factors and the GPDs. Since in the scalar diquark model, $\ell_{z}^{q}+s^{q}+\ell_{z}^{D}=1 / 2\left(\right.$ as $\left.s^{D}=0\right)$, the diquark contribution to the canonical OAM is $\ell_{z}^{D}=-0.484$ for the $u$ struck quark and $\ell_{z}=-0.016$ for the $d$ struck quark. The contributions of different partial waves to the quark OAM in LCCQM have been studied in [17].

The Wigner distributions for a longitudinally polarized quark in a longitudinally polarized proton, $\rho_{L L}^{q}\left(\mathbf{b}_{\perp}, \mathbf{p}_{\perp}\right)$, are shown in Fig. 8. Figure 8a, b represent $\rho_{L L}^{q}\left(\mathbf{b}_{\perp}, \mathbf{p}_{\perp}\right)$ in transverse momentum plane with fixed $\mathbf{b}_{\perp}=0.4 \mathrm{fm} \hat{y}$ and Fig. $8 \mathrm{c}, \mathrm{d}$ show the plots in the transverse impact parameter plane with $\mathbf{p}_{\perp}=0.3 \mathrm{GeV} \hat{y}$. The distributions are circularly symmetric for the $u$ and the $d$ quarks in the two planes. The circular symmetry implies that the $\rho_{L L}$ cannot contribute to the quark OAM as shown in Eq. (41). The picks of the distributions are at the center $(0,0)$ in the two planes. Therefore the quark polarization and the proton polarization tend to be parallel for $u$ and $d$ quarks. Figure 9 represents the distribution $\tilde{\rho}_{L L}^{q}\left(b_{x}, p_{y}\right)$ in a mixed transverse plane. The distributions are axially symmetric for both $u$ and $d$ quarks.

The distributions $\rho_{\Lambda \lambda}^{q}\left(\mathbf{b}_{\perp}, \mathbf{p}_{\perp}\right)$ are shown in Figs. 10 and 11 with the polarization of the proton $\Lambda=\uparrow$ and quark polarization $\lambda=\uparrow, \downarrow$ (Eq. (10)). Figure 10a-d represent the vari- 


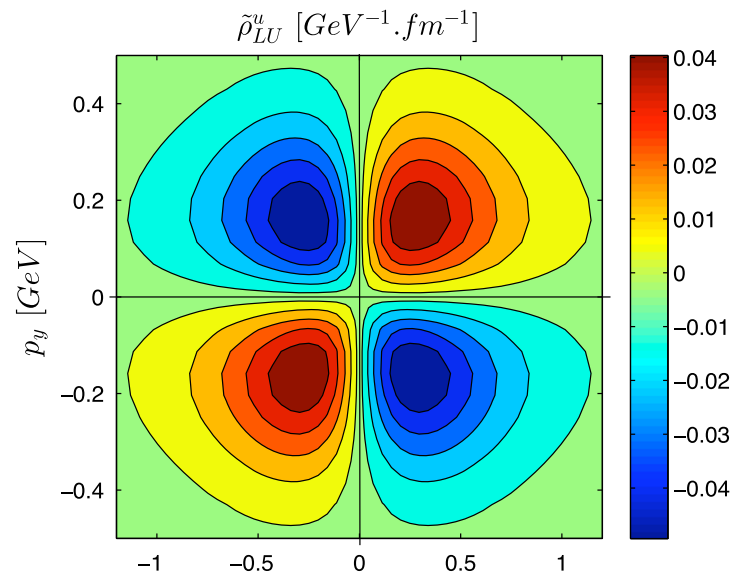

(a) (b)

$$
b_{x}[\mathrm{fm}]
$$

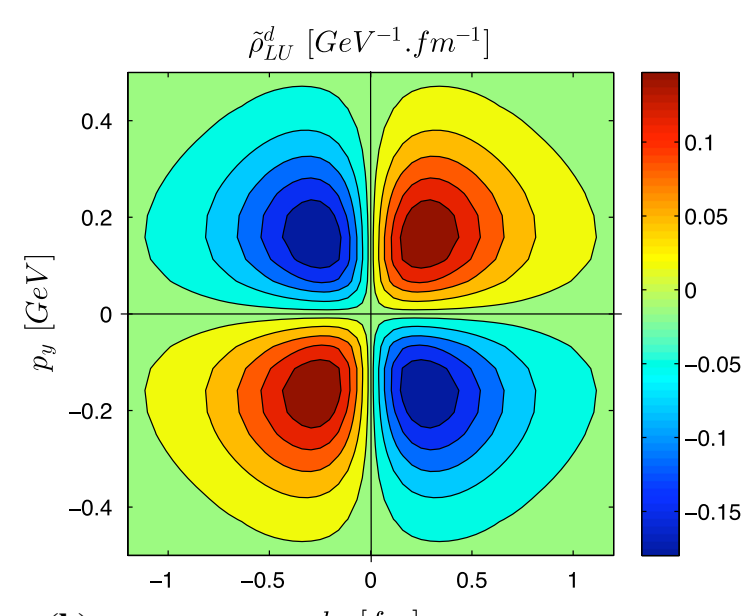

Fig. 7 The $\tilde{\rho}_{L U}^{q}\left(b_{x}, p_{y}\right)$ in the mixed transverse plane corresponding to the $u$ quark (a) and the $d$ quark (b)

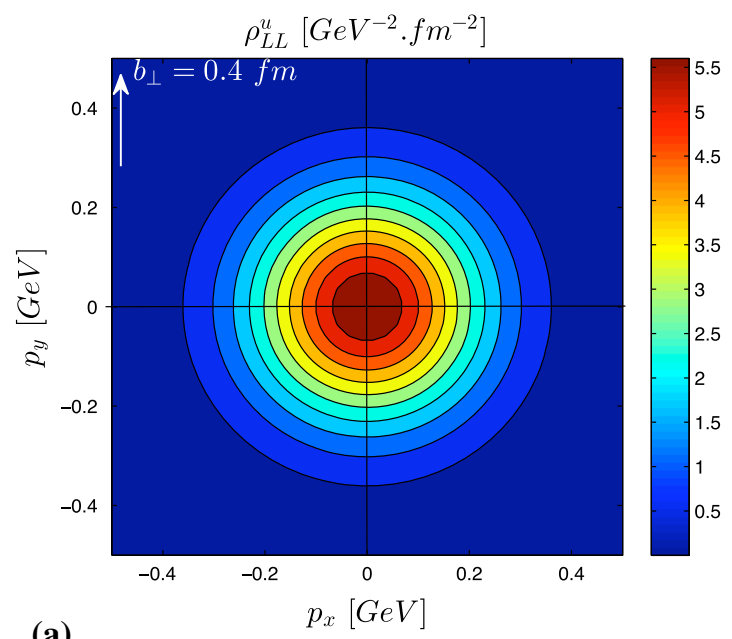

(a)

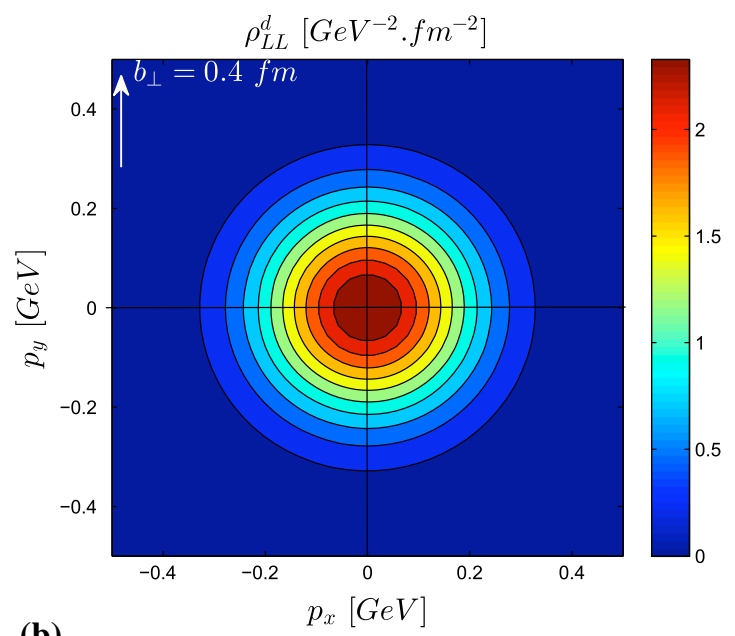

(b)

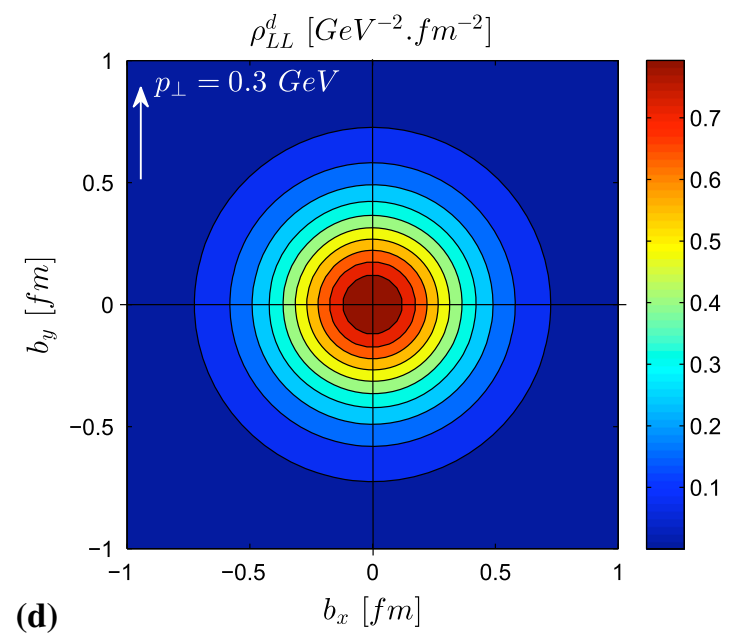

Fig. 8 The $\rho_{L L}^{q}\left(\mathbf{b}_{\perp}, \mathbf{p}_{\perp}\right)$ in the transverse momentum plane $(\mathbf{a}, \mathbf{b})$ with $\mathbf{b}_{\perp}=0.4 \hat{y} f m$ and in the transverse impact parameter plane (c, d) with $\mathbf{p}_{\perp}=0.3 \hat{y} \mathrm{GeV}$ for the $u$ and the $d$ quarks, respectively 


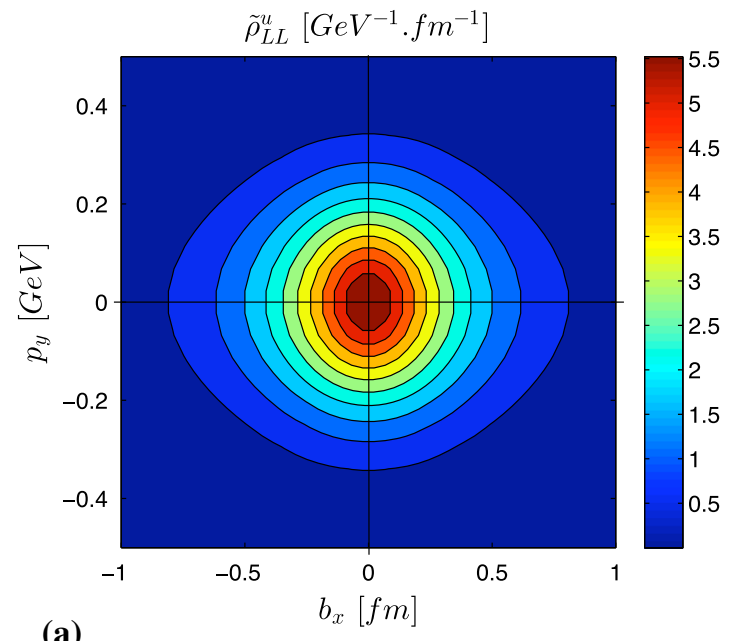

(a)

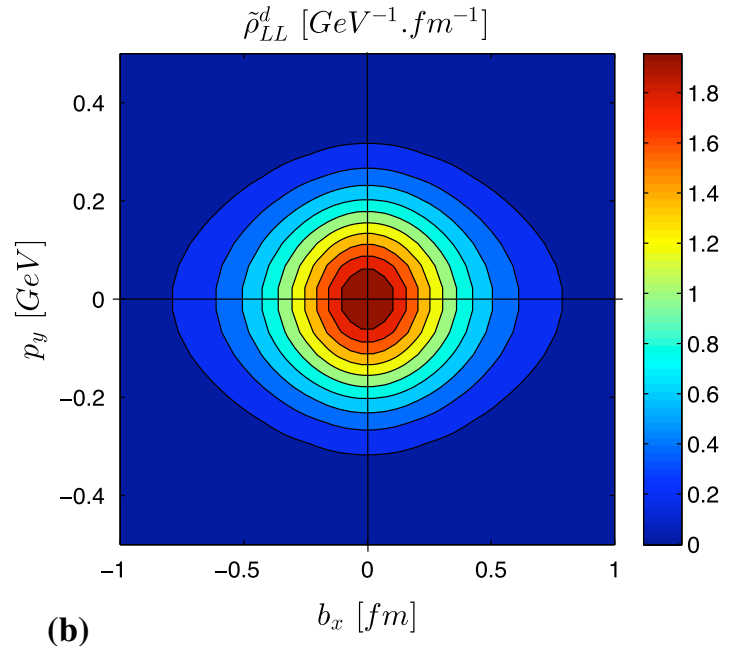

(b)

Fig. 9 The $\tilde{\rho}_{L L}^{q}\left(b_{x}, p_{y}\right)$ in mixed transverse plane for the $u$ and the $d$ quarks

ation of $\rho_{\Lambda \lambda}^{q}\left(\mathbf{b}_{\perp}, \mathbf{p}_{\perp}\right)$ in the transverse momentum plane for the $u$ and the $d$ quarks. We observe a circular symmetry for $\Lambda=\lambda$, but for $\Lambda \neq \lambda$ the distributions get distorted along $p_{x}$ for both $u$ and $d$ quarks. This is because in Eq. (9) the contributions from $\rho_{L U}$ and $\rho_{U L}\left(\rho_{L U}=-\rho_{U L}\right)$ get canceled for $\Lambda=\lambda$, whereas for $\Lambda \neq \lambda$ the contributions add up and cause the distortion.

We have shown the distributions for $\Lambda=\uparrow$, the other possible spin combinations in the transverse momentum plane can be found from $\rho_{\downarrow \lambda^{\prime}}^{q}\left(\mathbf{b}_{\perp}, p_{x}, p_{y}\right)=\rho_{\uparrow \lambda}^{q}\left(\mathbf{b}_{\perp},-p_{x}, p_{y}\right)$, where $\lambda^{\prime} \neq \lambda$. Figure $10 \mathrm{e}-\mathrm{h}$ show the variation of $\rho_{\Lambda \lambda}^{q}\left(\mathbf{b}_{\perp}, \mathbf{p}_{\perp}\right)$ in the transverse impact parameter plane for the $u$ and the $d$ quarks. The distributions are circularly symmetric in the transverse impact parameter space for $\Lambda=\lambda$ but the distributions get distorted for $\Lambda \neq \lambda$, due to the same reason as described in the case of the transverse momentum plane. Similar to the momentum space, the other possible spin combinations in the transverse impact parameter plane are found as $\rho_{\downarrow \lambda^{\prime}}^{q}\left(b_{x}, b_{y}, \mathbf{p}_{\perp}\right)=\rho_{\uparrow \lambda}^{q}\left(-b_{x}, b_{y}, \mathbf{p}_{\perp}\right)$, where $\lambda^{\prime} \neq \lambda$. The mixed transverse densities $\tilde{\rho}_{\Lambda \lambda}^{q}\left(b_{x}, p_{y}\right)$ are shown in Fig. 11 for the $u$ and the $d$ quarks. Again, for $\Lambda=\lambda$ the contribution from quadrupole distortions (Figs. 5, 7) $\tilde{\rho}_{U L}$ and $\tilde{\rho}_{L U}$ get canceled resulting from the axial symmetry but for $\Lambda \neq \lambda$ the contributions add up. The maxima of $\tilde{\rho}_{U U}$ and $\tilde{\rho}_{L L}$ are nearly equal (Figs. 3, 9). As a result, for $\Lambda \neq \lambda$, the destructive interference of these two distributions give almost zero at the center $\left(b_{x}=0, p_{y}=0\right)$ in Fig. $11 \mathrm{c}, \mathrm{d}$.

\section{Spin-spin and spin-OAM correlation}

In Figs. 4 and 6, we observe that the quark OAM tends to be anti-aligned with the quark spin and aligned to the proton spin for both $u$ and $d$ quarks. The correlation strength between proton spin and quark OAM is equal to the corre- lation between quark spin and quark OAM. Therefore, if the quark spin is parallel to the proton spin, i.e., $\Lambda=\uparrow, \lambda=\uparrow$, the contributions of $\rho_{U L}$ and $\rho_{L U}$ interfere destructively resulting from the circular symmetry for $u$ and $d$ quarks; see Figs. $10 \mathrm{a}, \mathrm{b}, \mathrm{e}, \mathrm{f}$. If the quark spin is anti-parallel to the proton spin, i.e., $\Lambda=\uparrow, \lambda=\downarrow$, the contributions of $\rho_{U L}$ and $\rho_{L U}$ interfere constructively resulting from a significant shift for $u$ and $d$ quarks; see Figs. 10c, d, g, h. One can notice that from Fig. 10 , the direction of the shift flips with the polarization flip when $\Lambda \neq \lambda$.

We compare our results with the light-cone constituent quark model (LCCQM) [11] and the light-cone spectator model [25] in Tables 3 and 4. The polarities of the $\rho_{U L}$ distributions are opposite to LCCQM but similar to the spectator model, whereas for $\rho_{L U}$, all the three models agree for the $u$ quark, but the agreement is lost for the $d$ quark. In our model, the average quadrupole distortion $Q_{b}^{i j}\left(\mathbf{p}_{\perp}\right)$ and $Q_{p}^{i j}\left(\mathbf{b}_{\perp}\right)$, in both the transverse momentum plane and the transverse impact parameter plane, are found to be zero, whereas a nonzero small quadrupole distortion is found in [11]. This may be due to the simple scalar diquark model considered here; inclusion of the axial vector diquark might improve the result. The quark OAM tends to be anti-aligned $\left(C_{z}^{u}<0, C_{z}^{d}<0\right)$ to the quark spin for both $u$ and $d$ quarks in our model, in LCCQM the quark OAM and quark spin tend to be aligned for both $u$ and $d$ quarks $\left(C_{z}^{u}>0, C_{z}^{d}>0\right)$. In our model, the quark OAM tends to be aligned to the proton spin for both $u$ and $d$ quarks $\left(\ell_{z}^{u}>0, \ell_{z}^{d}>0\right)$, whereas in [11], the quark OAM tends to be aligned $\left(\ell_{z}^{u}>0\right)$ to the proton spin for the $u$ quark and anti-aligned $\left(\ell_{z}^{d}<0\right)$ for $d$ quark. For a proton spin anti-aligned with the quark spin, the distributions $\rho_{\uparrow \downarrow}^{q}$ for both $u$ and $d$ quarks show a stronger dipolar structure in our model compared to the LCCQM. QCD or some model independent calculations are required to resolve the differences. 
Fig. 10 The $\rho_{\Lambda \lambda}^{q}\left(\mathbf{b}_{\perp}, \mathbf{p}_{\perp}\right)$ for $\Lambda=\uparrow$ and $\lambda=\uparrow, \downarrow$ in transverse momentum plane (a-d) with $\mathbf{b}_{\perp}=0.4 \hat{y} \mathrm{fm}$ and in transverse impact parameter plane (e-h) with $\mathbf{p}_{\perp}=0.3 \hat{y} \mathrm{GeV}$ for $u$ and $d$ quarks

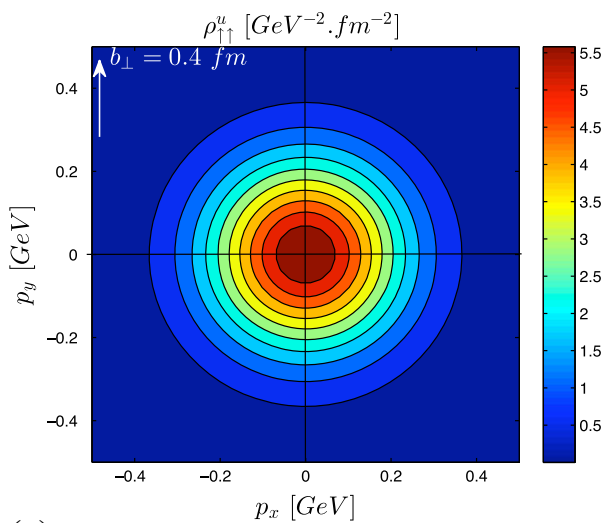

(a)
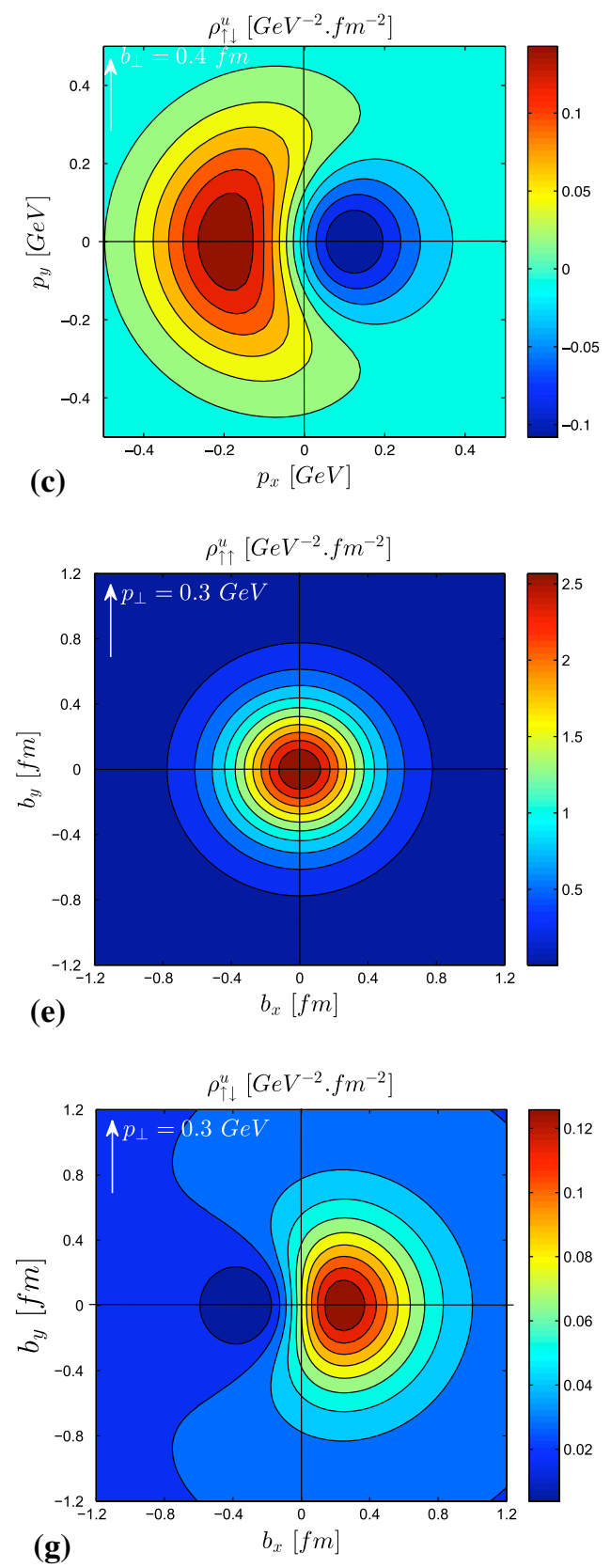

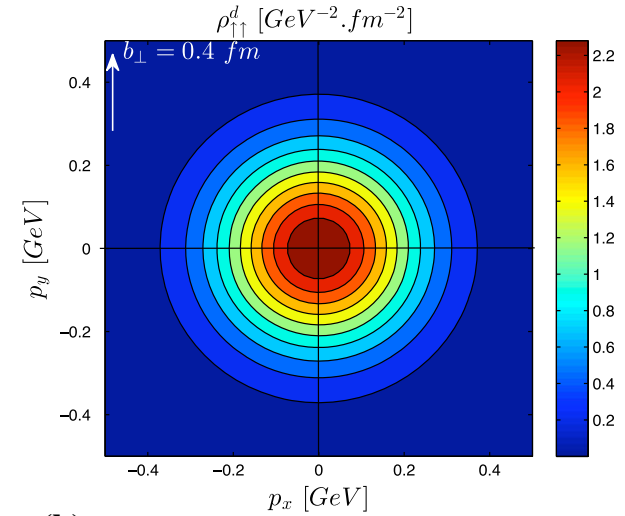

(b)
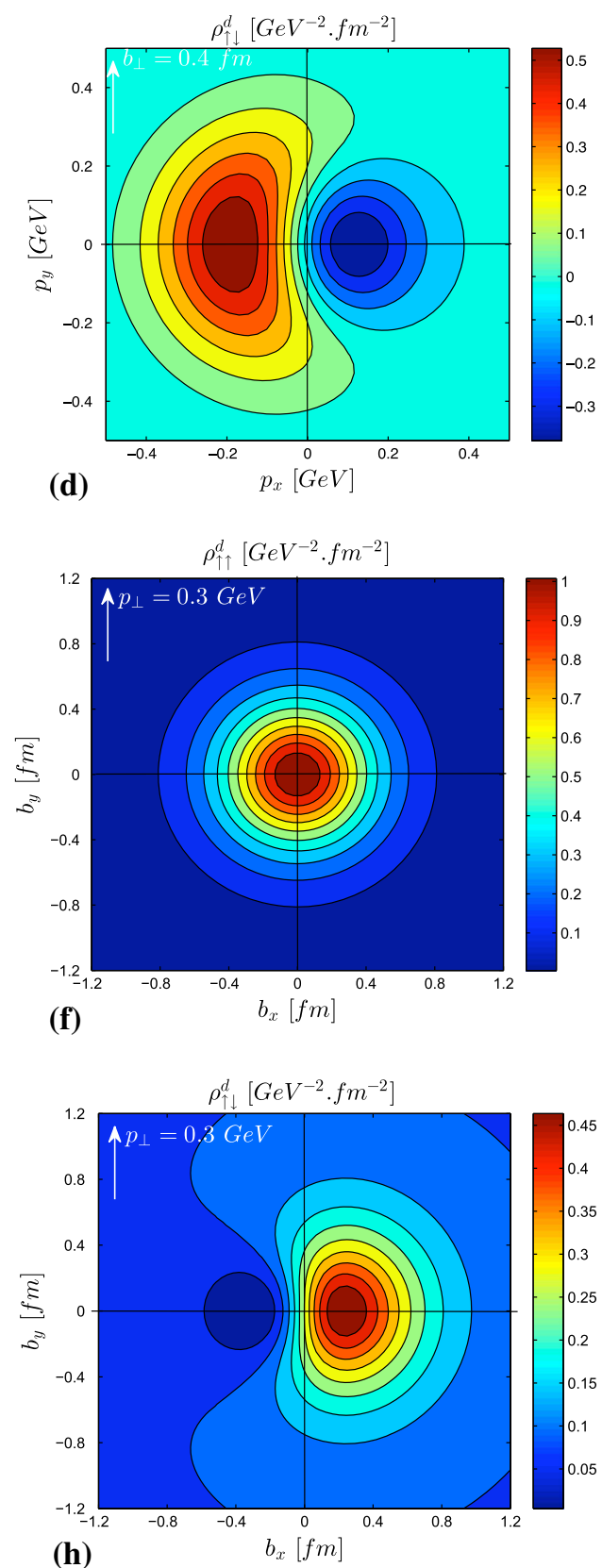

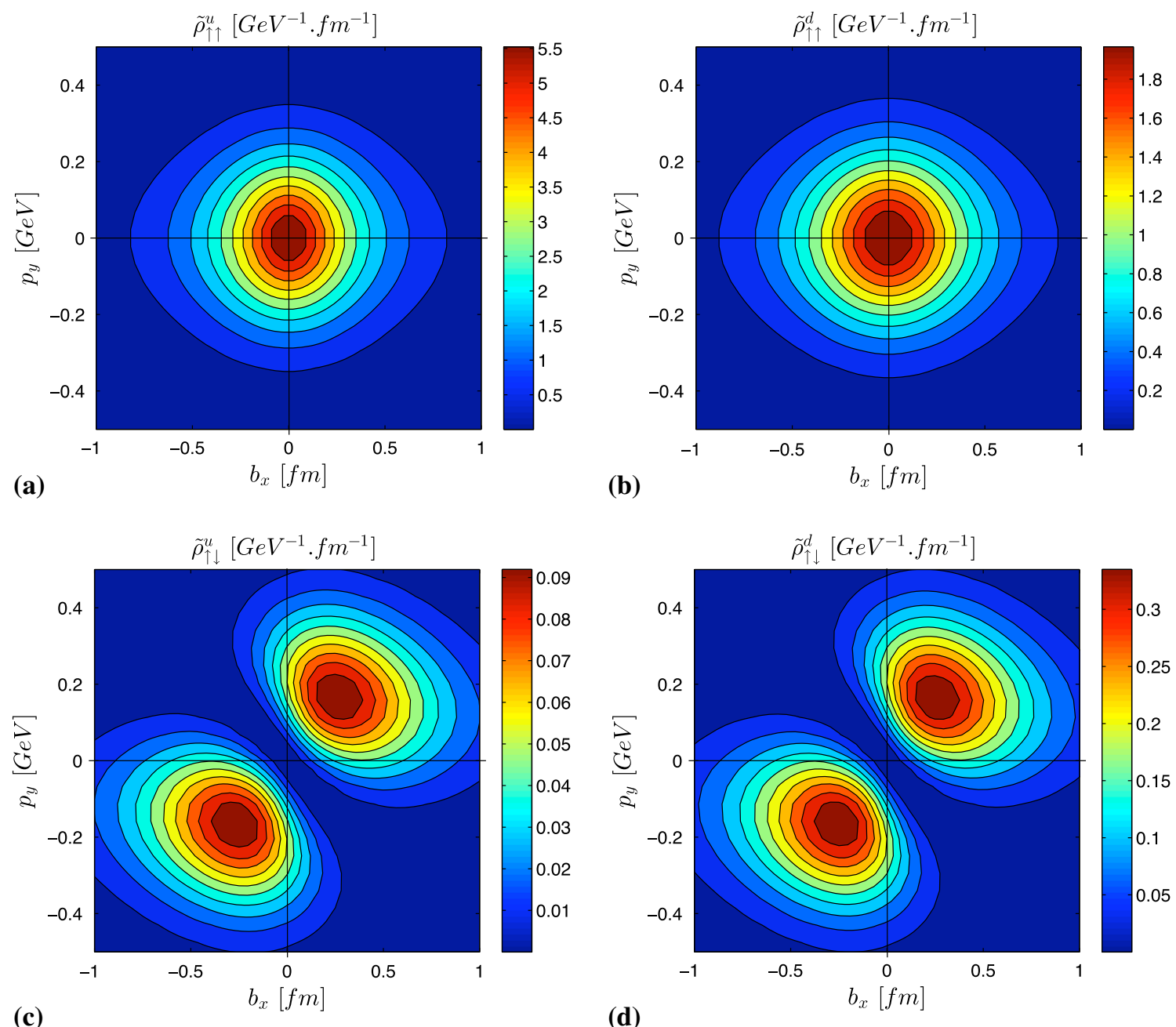

Fig. 11 The $\tilde{\rho}_{\Lambda \lambda}^{q}\left(b_{x}, p_{y}\right)$ for $\Lambda=\uparrow$ and $\lambda=\uparrow, \downarrow$ in mixed transverse plane for the $u$ and the $d$ quarks

Table 3 Comparison of $\rho_{U L}$ in different models in momentum space (left panel) and in impact parameter space (right panel). + -/ + represent the polarity of the dipolar distributions and the maxima of the distributions are given within the bracket

\begin{tabular}{lccccccc}
\hline$\rho_{U L}\left(\mathbf{p}_{\perp}\right)$ & Our model & Ref. [11] & Ref. [25] & $\rho_{U L}\left(\mathbf{b}_{\perp}\right)$ & Our model & Ref. [11] & Ref. [25] \\
\hline$u$ & $-+(0.1)$ & $+-(0.6)$ & $-+(0.010)$ & $u$ & $+-(0.06)$ & $-+(0.5)$ & $+-(0.010)$ \\
$d$ & $-+(0.3)$ & $+-(0.6)$ & $-+(0.005)$ & $d$ & $+-(0.2)$ & $-+(0.5)$ & $+-(0.005)$ \\
\hline
\end{tabular}

Table 4 Comparison of $\rho_{L U}$ in different models in momentum space (left panel) and in impact parameter space (right panel). + -/ + represent the polarity of the dipolar distributions and the maxima of the distributions are given within the bracket

\begin{tabular}{llllllll}
\hline$\rho_{L U}\left(\mathbf{p}_{\perp}\right)$ & Our model & Ref. [11] & Ref. [25] & $\rho_{L U}\left(\mathbf{b}_{\perp}\right)$ & Our model & Ref. [11] & Ref. [25] \\
\hline$u$ & $+-(0.1)$ & $+-(0.35)$ & $+-(0.010)$ & $u$ & $-+(0.06)$ & $-+(0.3)$ & $-+(0.005)$ \\
$d$ & $+-(0.3)$ & $-+(0.03)$ & $-+(0.002)$ & $d$ & $-+(0.2)$ & $+-(0.015)$ & $+-(0.0005)$ \\
\hline
\end{tabular}

\section{GTMDs}

At leading twist, there are 16 GMDs. The variation of GTMDs (Eqs. (53-56)) for the $u$ and the $d$ quarks are shown in Fig. 12. The left column is for different values of $\Delta_{\perp}^{2}$ with a fixed $\mathbf{p}_{\perp}=0.3 \mathrm{GeV}$ and the right column is for different values of $p_{\perp}$ with a fixed $\Delta_{\perp}^{2}=1.0 \mathrm{GeV}^{2}$. We observe that the peaks of the distributions decrease with increasing $\Delta_{\perp}$ and shift toward higher $x$. Thus, the distributions $F_{1,1}^{q}, F_{1,4}^{q}, G_{1,1}^{q}, G_{1,4}^{q}$, having a quark with fixed transverse 

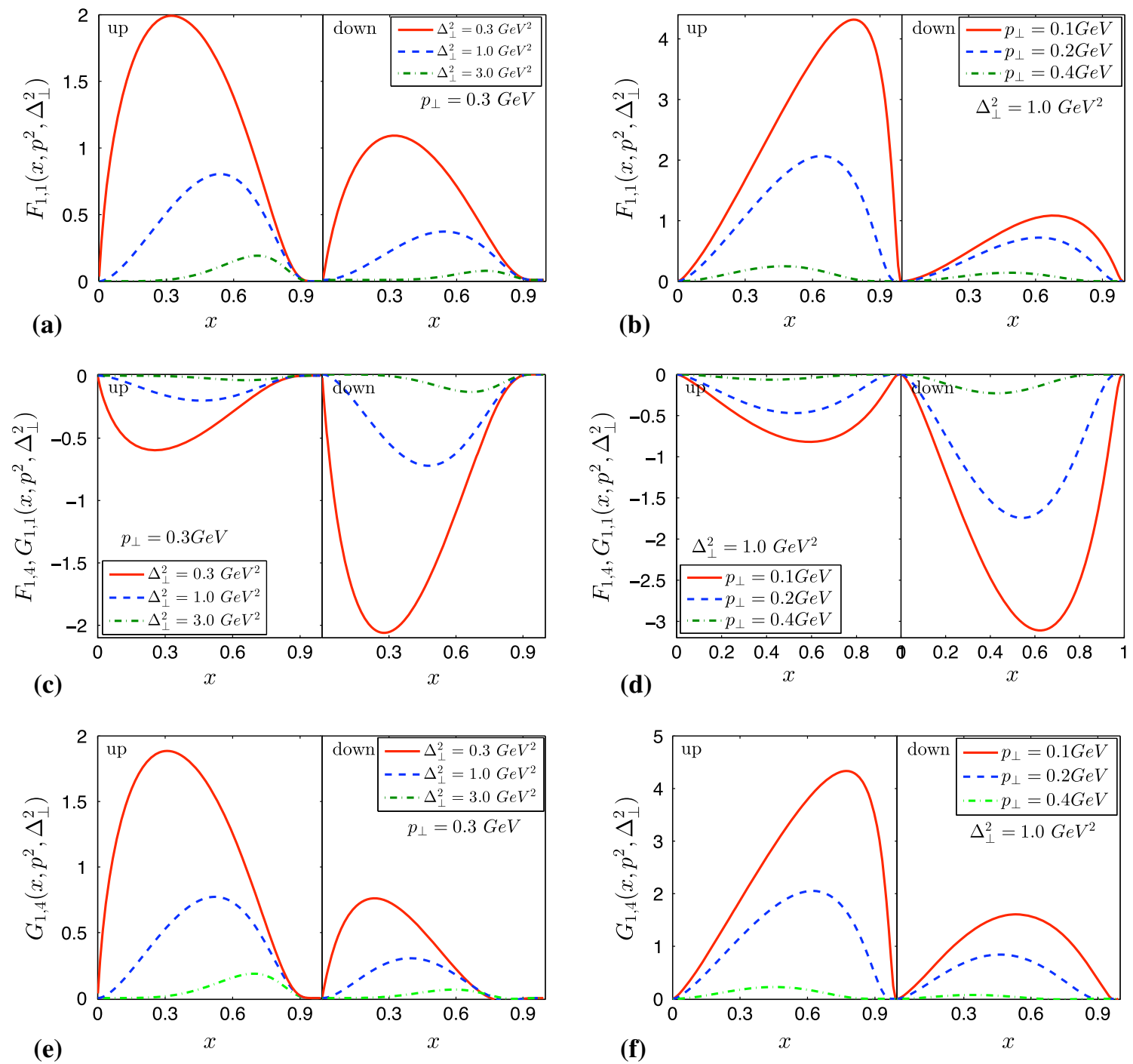

Fig. 12 GTMDs as functions of $x$ for both $u$ and $d$ quarks at different fixed values of $\mathbf{p}_{\perp}$ and $\Delta_{\perp}$

$\mathbf{p}_{\perp}$, highly depend on the momentum transfer $\Delta_{\perp}$ between initial and final proton. The behavior of $F_{1,1}$ for $u$ and $d$ quarks are almost the same except in magnitude which is larger for the $u$ quark than the $d$ quark. In $F_{1,4}\left(=G_{1,1}\right)$, the maximum for the $d$ quark is greater than the maximum for the $u$ quark and opposite to $F_{1,1}$ and $G_{1,4}$. The GTMDs as functions of $x$ are shown in the right column of Fig. 12 for the different values of $\mathbf{p}_{\perp}$ with a fixed value of $\Delta_{\perp}^{2}=1.0 \mathrm{GeV}^{2}$. In this case, the peaks of the distributions shift toward lower $x$ and decrease as $\mathbf{p}_{\perp}$ increases.

\section{Conclusions}

We have calculated the Wigner distributions in a quark-scalar diquark model of the proton. We have used the light-front wave functions for the state that are predicted by the softwall ADS/QCD. The Wigner distributions of both unpo- larized quark in unpolarized proton as well as the distortions in momentum and position space due to the polarization of the quark/proton are calculated. The results are compared and contrasted with other model estimates, in particular with those models that assume a confining potential. The Wigner functions are related to GTMDs that give information on the canonical OAM as well as the spin-orbit correlation of the quarks. The kinetic OAM can be calculated in terms of the GPDs in this model. We have calculated both the canonical and the kinetic OAM and compared with other model calculations. In our case the proton state consists of an active quark which can be either a $u$ or a $d$ quark, and a scalar diquark. So the sum of the OAM of the $u$ and the $d$ quark is not expected to be the same. In fact the kinetic and canonical OAM of the $u$ quark are positive in this model, whereas those of the $d$ quark are negative. We have also calculated the pretzelosity in this model using a model-dependent relation. As $x \rightarrow 1$ the difference between 
kinetic and canonical OAM vanishes as all the momentum is carried by the active quark. Further work would involve a calculation of the Wigner distributions incorporating transverse polarization.

Open Access This article is distributed under the terms of the Creative Commons Attribution 4.0 International License (http://creativecomm ons.org/licenses/by/4.0/), which permits unrestricted use, distribution, and reproduction in any medium, provided you give appropriate credit to the original author(s) and the source, provide a link to the Creative Commons license, and indicate if changes were made. Funded by SCOAP ${ }^{3}$.

\section{References}

1. X. Ji, Phys. Rev. D 55, 7114 (1997)

2. A.V. Radyushkin, Phys. Rev. D 56, 5524 (1997)

3. K. Goeke, M.V. Polyakov, M. Vanderhagen, Prog. Part. Nucl. Phys. 47, 401 (2001)

4. M. Diehl, Phys. Rept. 388, 41 (2003)

5. J.C. Collins, D.E. Soper, Nucl. Phys. B 193, 381 (1981)

6. D. Sivers, Phys. Rev. D 41, 83 (1990)

7. P.J. Mulders, R.D. Tangerman, Nucl. Phys. B 461, 197 (1996)

8. D. Boer, P.J. Mulders, Phys. Rev. D 57, 5780 (1998)

9. X. Ji, Phys. Rev. Lett. 91, 062001 (2003)

10. A.V. Belitsky, X. Ji, F. Yuan, Phys. Rev. D 69, 074014 (2004)

11. C. Lorce, B. Pasquini, Phys. Rev. D 84, 014015 (2011)

12. S. Boffi, B. Pasquini, M. Traini, Nucl. Phys. B 649, 243 (2003)

13. S. Boffi, B. Pasquini, M. Traini, Nucl. Phys. B 680, 147 (2004)

14. B. Pasquini, M. Pincetti, S. Boffi, Phys. Rev. D 72, 094029 (2005)

15. B. Pasquini, M. Pincetti, S. Boffi, Phys. Rev. D 76, 034020 (2007)

16. B. Pasquini, S. Cazzaniga, S. Boffi, Phys. Rev. D 78, 034025 (2008)

17. C. Lorce, B. Pasquini, X. Xiong, F. Yuan, Phys. Rev. D 85, 114004 (2012)

18. C. Lorce, Phys. Rev. D 74, 054019 (2006)

19. C. Lorce, Phys. Rev. D 78, 034001 (2008)
20. C. Lorce, Phys. Rev. D 79, 074027 (2009)

21. V.Y. Petrov, M.V. Polyakov. arXiv:hep-ph/0307077

22. D. Diakonov, V. Petrov, Phys. Rev. D 72, 074009 (2005)

23. A. Mukherjee, S. Nair, V.K. Ojha, Phys. Rev. D 90(1), 014024 (2014)

24. A. Mukherjee, S. Nair, V.K. Ojha, Phys. Rev. D 91(5), 054018 (2015)

25. Tianbo Liu, Bo-Qiang Ma, Phys. Rev. D 91, 034019 (2015)

26. T. Gutsche, V.E. Lyubovitskij, I. Schmidt, A. Vega, Phy. Rev. D 89, 054033 (2014)

27. C. Mondal, D. Chakrabarti, Eur. Phys. J. C 75, 261 (2015)

28. S.J. Brodsky, G.F. de Téramond. arXiv:1203.4025 [hep-ph]

29. D. Chakrabarti, C. Mondal, Phys. Rev. D 92, 074012 (2015)

30. T. Maji, C. Mondal, D. Chakrabarti, O.V. Teryaev, JHEP 1601, 165 (2016)

31. R. Jakob, P.J. Mulders, J. Rodrigues, Nucl. Phys. A 626, 937 (1997)

32. S. Meissner, A. Metz, M. Schlegel, K. Goeke, JHEP 0808, 038 (2008)

33. S. Meissner, A. Metz, M. Schlegel, JHEP 0908, 056 (2009)

34. M.G. Echevarria, A. Idilbi, K. Kanazawa, C. Lorcé, A. Metz, B. Pasquini, M. Schlegel, Phys. Lett. B 759, 336 (2016). doi:10.1016/ j.physletb.2016.05.086

35. R.I. Jaffe, A. Manohar, Nucl. Phys. B 337, 509 (1990)

36. X.D. Ji, Phys. Rev. Lett. 78, 610 (1997)

37. X.S. Chen, X.F. Lu, W.M. Sun, F. Wang, T. Goldman, Phys. Rev. Lett. 100, 232002 (2008)

38. K.F. Liu, C. Lorc, Eur. Phys. J. A 52(6), 160 (2016)

39. K. Kanazawa, C. Lorce, A. Metz, B. Pasquini, M. Schlegel, Phy. Rev. D 90, 014028 (2014)

40. A. Becchetta, F. Conti, M. Radici, Phys. Rev. D 78, 074010 (2008)

41. T. Gutsche, V.E. Lyubovitskij, I. Schmidt, A. Vega, Phys. Rev. D 91, 054028 (2015)

42. D. de Florian, R. Sassot, M. Stratmann, W. Vogelsang, Phys. Rev. D 80, 034030 (2009)

43. E.R. Nocera et al. (NNPDF collaboration), Nucl. Phy. B 887, 276 (2014)

44. E.R. Nocera, Phys. Lett. B 742, 117 (2015)

45. H. Avakian, S.J. Brodsky, A. Deur, F. Yuan, Phys. Rev. Lett. 99, 082001 (2007)

46. F. Close, W. Melnitchouk, Phys. Rev. C 68, 035210 (2003) 